\title{
Regge behaviors in orbitally excited spectroscopy of charmed and bottom baryons
}

\author{
Duojie Jia $\odot,{ }^{1,2, *}$ Wen-Nian Liu, ${ }^{1}$ and Atsushi Hosaka ${ }^{2}$ \\ ${ }^{1}$ Institute of Theoretical Physics, College of Physics and Electronic Engineering, \\ Northwest Normal University, Lanzhou 730070, China \\ ${ }^{2}$ Research Center for Nuclear Physics, Osaka University, \\ 10-1 Mihogaoka, Ibaraki, Osaka 567-0047, Japan
}

(Received 21 July 2019; accepted 9 December 2019; published 18 February 2020)

\begin{abstract}
Stimulated by recent progress made by the LHCb Collaboration in discoveries of new bottom baryons, e.g., the $\Xi_{b}(6227)^{-}$and the $\Sigma_{b}(6097)^{ \pm}$, we reexamine the orbitally excited spectrum of the charmed and bottom baryons using the Regge approach in the heavy quark-diquark picture. The results indicate that the spin-averaged mass spectrum of the orbitally excited charmed and bottom baryons can be described by a linear Regge relation, which is derived from the rotating QCD string model. By giving further masssplitting analysis of spin-dependent interactions, we explain the baryons $\Xi_{b}(6227)^{-}$and the $\Sigma_{b}(6097)^{ \pm}$, and the $\Sigma_{c}(2800)$ and $\Xi_{c}^{\prime}(2930)$ to be the $1 P$-wave baryons, all with the spin-parity $J^{P}=3 / 2^{-}$preferably. Mass prediction of the bottom baryon $\Xi_{b}$ in its $P$-wave- and $D$-waves are presented, providing clues for the coming experiments like the $\mathrm{LHCb}$ to find them.
\end{abstract}

DOI: 10.1103/PhysRevD.101.034016

\section{INTRODUCTION}

The information from singly heavy ( $\mathrm{SH}$ ) baryon spectrum is quite helpful to deepen the understanding of nonperturbative quantum chromodynamics (QCD) as the heavy quark inside a baryon endows us with a "flavor tag" to look further into the nature of the interquark forces [1]. This forms an analogy with the hydrogenlike atoms, in which the quantum states of the binding systems are labeled well by that of light degrees of freedom, and provides us with a good chance to further construct excited states of the bottom baryon family. In the past two decades, more than twenty charmed baryon candidates have been announced by the different experimental collaborations [2]. Differing from the charmed baryons, searching for the bottom baryon states is far from easy for experiment since the bottom baryons seems to be more difficult to produce than the charmed baryons. Only several bottom baryon candidates were reported before 2017 .

Recently, the LHCb Collaboration discovered, one after another, two new bottom baryon states, the $\Xi_{b}(6227)^{-}$by studying its final states of the $\Lambda_{b}^{0} K^{-}$and $\Xi_{b}^{0} \pi^{-}$[3], and the $\Sigma_{b}(6097)^{ \pm}$in the final states of $\Lambda_{b}^{0} \pi^{ \pm}$[4]. The two baryons differ about $130 \mathrm{MeV}$ in mass, close to the mass difference

\footnotetext{
*jiadj@nwnu.edu.cn
}

Published by the American Physical Society under the terms of the Creative Commons Attribution 4.0 International license. Further distribution of this work must maintain attribution to the author(s) and the published article's title, journal citation, and DOI. Funded by SCOAP. of $u / d$ and $s$ constituent quarks, and exhibit similar decay behaviors in width and final states. After their observations, the two baryons become the key states for establishing the whole family of the excited bottom baryons, and their properties and inner structures become hot topics for the heavy baryon study [5-11]. Most of these studies prefer to suggest that the $\Xi_{b}(6227)^{-}$is the $P$-wave strange partner of the $\Sigma_{b}(6097)^{ \pm}$, with spin-parity $J^{P}=3 / 2^{-}$or $5 / 2^{-}$while there are also the molecular accommodation [12]. For more discussions on the excited heavy baryons, see Refs. [13-20].

To further decode inner structure of the $\Xi_{b}(6227)^{-}$and $\Sigma_{b}(6097)^{ \pm}$, it becomes important to explore the charmed and bottom baryons in a coherent way so that these newly discovered baryons can be analysed in connection with the existing SH baryons in the charm sector, e.g., the $\Sigma_{c}(2800)$ and $\Xi_{c}^{\prime}(2930)$, whose quantum numbers are not established experimentally yet $[2,21,22]$. Obviously, it will be helpful to deepen our understanding of nonperturbative QCD if some analogies were found among these $\mathrm{SH}$ hadron systems. For the two charmed baryons mentioned above, quark models predict their quantum number to be [17-20],

$$
\begin{aligned}
& J^{P}\left(\Sigma_{c}(2800)\right)=\frac{1^{-}}{2} \quad \text { or } \quad \frac{3}{2}^{-}, \\
& J^{P}\left(\Xi_{c}^{\prime}(2930)\right)=\frac{3^{-}}{2} \text { or } \quad \frac{5}{2}^{-} .
\end{aligned}
$$

Based on the Regge trajectories, authors of Ref. [23] assign the $\Sigma_{c}(2800)$ and $\Xi_{c}^{\prime}(2930)$ to be the $P$-wave states with $J^{P}=3 / 2^{-}$. 
The purpose of this work is to explore the orbitally excited mass spectrum of the $\mathrm{SH}$ baryons $q q Q$ with the help of Regge trajectory and accommodate the newly discovered bottom baryons $\Xi_{b}(6227)$, the $\Sigma_{b}(6097)^{ \pm}$, as well as the charmed baryons $\Sigma_{c}(2800)$ and $\Xi_{c}^{\prime}(2930)$. Our mass analysis indicates that a linear Regge trajectory is able to describe the spin-averaged mass spectrum of the existing singly heavy baryons in their orbitally excited states. Further mass-splitting analysis of the orbitally excited SH baryons suggests that the quantum numbers of the four SH baryons considered here are $J^{P}=3 / 2^{-}$preferably. We also make the mass prediction of the bottom baryon $\Xi_{b}$ in its $P$-wave- and $D$-waves, which should be tested by future experiments, especially the $\mathrm{LHCb}$.

The paper is organized as follows. After the introduction, we show how the linear Regge relation can be employed to demonstrate Regge behaviors in the spin-averaged masses of orbitally excited SH baryons in Sec. II, where the trajectory parameters are discussed. In Sec. III, we perform mass calculations for the orbital excitations of the $\mathrm{SH}$ baryons with $L=0,1$ and $2\left(\Lambda_{c}, \Xi_{c}\right.$ cases $)$ by including the spin-dependent interactions, and assign the $J^{P}$ quantum number for the $\Sigma_{c}(2800)$ and the $\Xi_{c}^{\prime}(2930)$. In Sec. IV the similar analysis is applied to the singly bottom baryons and to assign the quantum number for the newly reported baryons $\Xi_{b}(6227)$ and the $\Sigma_{b}(6097)^{ \pm}$. The masses of the bottom baryon states $\Xi_{b}$ in the $P$-wave- and $D$-waves are also predicted. Finally, the paper ends with the discussions and summary in Sec. V.

\section{SPIN-INDEPENDENT MASSES FOR SINGLY HEAVY BARYONS}

The notion of Regge trajectory or Regge mass relation $L=\alpha^{\prime} M^{2}+\alpha_{0}$ for hadrons, which connects high energy scattering and the spectrum of hadrons in general theory [24], provides us a powerful way to organize the whole spectrum of a given family of the hadrons. In the case of light hadrons, the Regge trajectories are commonly believed to be linear and parallel, and these features are also tested to be true roughly for the SH hadrons in Ref. [25] provided that the hadron mass undergoes a shift $M \rightarrow M-M_{Q}$, with $M_{Q}$ the heavy quark mass. As such, one can estimate the spin-averaged masses of $\Sigma_{Q}$ and $\Xi_{Q}^{\prime}$ in their orbitally excited states with the help of the trajectory information of the partner SH baryons or mesons.

We begin with a linear Regge relation [26,27], which is derived from the rotating string model (see Appendix A),

$$
\left(M-M_{Q}\right)^{2}=\pi a L+\left(m_{d}+\frac{P_{Q}^{2}}{M_{Q}}\right)^{2},
$$

This relation connects the shifted mass of a SH baryon squared, $\left(M-M_{Q}\right)^{2}$, with its quantum number of orbital angular momentum $L$. Here, $a$ stands for the tension of the string connected to the heavy quark with mass $M_{Q}$ at one end and the light diquark with mass $m_{d}$ at the other, and $M$ is the mass of the heavy baryon. In Eq. (2), the intercept depends on the diquark mass $m_{d}$ and the nonrelativistic kinematic energy $P_{Q}^{2} / M_{Q}$ of the heavy quark through $a_{0}=\left(m_{d}+P_{Q}^{2} / M_{Q}\right)^{2}$, with

$$
P_{Q} \equiv M_{Q} v_{Q} \simeq M_{Q}\left(1-\frac{m_{\text {bare } Q}^{2}}{M_{Q}^{2}}\right)^{1 / 2}
$$

where $m_{\text {bare } Q}=1.275 \mathrm{GeV}$ and $4.18 \mathrm{GeV}$ are the bare masses of the heavy charmed quark $Q=c$ and bottom quark $Q=b$, respectively. Here, $v_{Q}=\left(1-\frac{m_{\text {bare }}^{2}}{M_{Q}^{2}}\right)^{1 / 2}$ stands for the velocity of the heavy quark, which is conserved in the heavy quark limit.

The simplest way to understand Eq. (2) is to view it as an extension of the Selem-Wilczek relation [28] for the SH hadrons,

$$
M-M_{Q}=\sqrt{\frac{\alpha}{2} L}+2^{1 / 4} \kappa \frac{\mu^{3 / 2}}{L^{1 / 4}},
$$

which scales as $\left(M-M_{Q}\right)^{2} \propto(\alpha / 2) L$ when $L \rightarrow \infty$ but divergent at $L=0$. As $L \gg 1$, Eq. (2) reduces to Eq. (4), $M-M_{Q}=\sqrt{\pi a L}+a_{0} /(2 \sqrt{\pi a L})$, while it is finite at $L=0$ and becomes

$$
M(1 S)=M_{Q}+m_{d}+\frac{P_{Q}^{2}}{M_{Q}} .
$$

This is consistent with the prediction by the heavy quark symmetry for the ground state $(L=0)$ [29] as $M_{Q}$ is very close to the bare mass of the heavy quark $Q$ when $Q$ is heavy, as indicated by Eq. (A3) in Appendix A. The heavy quark limit defined by $m_{\text {bare } Q} \rightarrow \infty$ implies $M_{Q} \simeq$ $m_{\text {bare } Q} \rightarrow \infty$ and the 3 -velocity of heavy quark $v_{Q} \rightarrow 0$ (see Appendix A). Here, the $m_{d}$ plays the role of the energy of the light brown muck in a baryon in the lowest states. We note that the orbital angular momentum $L$ here corresponds to the $\lambda$ mode in the quark model [30,31].

In the light of the rotating string model [28], one can view the heavy baryon mass $M$ as the sum of the mass $M_{Q}$ of heavy quark at one end of the string and the string energy $\sqrt{\pi a L+a_{0}}$, with the light diquark tied to the other. We note that the full quantum treatment $[32,33]$ of the QCD string provides merely an additional constant $\alpha_{0}$ added to $L$ as expected by the Regge trajectory of hadrons.

As will be seen in what follows, the mass of the diquark $(q q)_{d}$ in Eq. (2) varies when the strangeness and its spin involve. In a SH baryon with zero strangeness, the diquark can be either of scalar $(\operatorname{spin}=0)$ or of vector $(\operatorname{spin}=1)$, 
TABLE I. The observed quantum numbers and masses (in MeV) [2] of the charmed and charm-strange baryons $\Lambda_{c}$ and $\Xi_{c}^{0}$ that contain the scalar-diquark. The $J^{P}$ of some states indicated by the question marks are the quark-model predictions [26], and have not been established experimentally. The errors less than $5 \mathrm{MeV}$ are not indicated.

\begin{tabular}{|c|c|c|c|c|c|c|}
\hline States, $J^{P}$ & Baryon & Mass & This work & Baryon & Mass & This work \\
\hline $1^{2} S_{1 / 2}, 1 / 2^{+}$ & $\Lambda_{c}^{+}$ & $2286.46(14)$ & 2286.0 & $\Xi_{c}^{+}$ & $2467.87(30)$ & 2469.1 \\
\hline $1^{2} P_{1 / 2}, 1 / 2^{-}$ & $\Lambda_{c}(2595)^{+}$ & $2592.25(28)$ & 2588.7 & $\Xi_{c}(2790)^{+}$ & $2792.0(5)$ & 2778.6 \\
\hline $1^{2} P_{3 / 2}, 3 / 2^{-}$ & $\Lambda_{c}(2625)^{+}$ & 2628.11(19) & 2628.9 & $\Xi_{c}(2815)^{+}$ & $2816.67(31)$ & 2816.5 \\
\hline $1^{2} D_{3 / 2}, 3 / 2^{+}$ & $\Lambda_{c}(2860)^{+}$ & $2856.1_{-6.0}^{+2.3}$ & 2857.3 & $\Xi_{c}(3055)^{+}$ & $3055.9(4)$ & 3058.7 \\
\hline $1^{2} D_{5 / 2}, 5 / 2^{+}$ & $\Lambda_{c}(2880)^{+}$ & $2881.63(24)$ & 2880.2 & $\Xi_{c}(3080)^{+}$ & $3077.2(4)$ & 3079.7 \\
\hline $1^{2} S_{1 / 2}, 1 / 2^{+}$ & $\Lambda_{b}^{0}$ & $5619.60(17)$ & 5615.5 & $\Xi_{b}$ & $5791.9(5)$ & 5792 \\
\hline $1^{2} P_{1 / 2}, 1 / 2^{-}$ & $\Lambda_{b}(5912)^{0}$ & $5912.20(21)$ & 5908.5 & $\Xi_{b}$ & & 6116.9 \\
\hline $1^{2} P_{3 / 2}, 3 / 2^{-}$ & $\Lambda_{b}(5920)^{0}$ & $5919.92(19)$ & 5921.4 & $\Xi_{b}$ & & 6129.1 \\
\hline $1^{2} D_{3 / 2}, 3 / 2^{+}$ & $\Lambda_{b}(6146)^{0}$ & 6146.17 & 6144.8 & $\Xi_{b}$ & & 6376.9 \\
\hline $1^{2} D_{5 / 2}, 5 / 2^{+}$ & $\Lambda_{b}(6152)^{0}$ & 6152.51 & 6152.2 & $\Xi_{b}$ & & 6383.6 \\
\hline
\end{tabular}

TABLE II. The observed quantum numbers and masses (in MeV) [2] charmed and charm-strange baryons that contain vector-diquark. The $J^{P}$ of some states indicated by the question marks are the quark-model predictions.

\begin{tabular}{|c|c|c|c|c|c|c|}
\hline States, $J^{P}$ & Baryon & Mass & This work & Baryon & Mass & This work \\
\hline $1^{2} S_{1 / 2}, 1 / 2^{+}$ & $\Sigma_{c}(2455)^{++}$ & $2453.97(14)$ & 2452.7 & $\Xi_{c}^{\prime}$ & 2578.8(5) & 2586.0 \\
\hline $1^{4} S_{3 / 2}, 3 / 2^{+}$ & $\Sigma_{c}(2520)^{++}$ & $2518.41_{-0.19}^{+0.21}$ & 2517.8 & $\Xi_{c}^{\prime 0}(2645)$ & $2646.32(31)$ & 2641.6 \\
\hline $1^{2 J+1} P_{J}, ? ?$ & $\Sigma_{c}(2800)^{++} ?$ & $2801_{-6}^{+4}$ & $\ldots$ & $\Xi_{c}^{\prime}(2930) ?$ & 2931(6) & $\ldots$ \\
\hline $1^{2} S_{1 / 2}, 1 / 2^{+}$ & $\Sigma_{b}^{+}$ & 5811.3(1.9) & 5811.0 & $\Xi_{b}^{\prime}(5935)^{-}$ & $5935.02(05)$ & 5937.1 \\
\hline $1^{4} S_{3 / 2}, 3 / 2^{+}$ & $\Sigma_{b}^{*+}$ & 5832.1(1.9) & 5832.0 & $\Xi_{b}^{\prime}(5955)$ & $5955.33(13)$ & 5955.0 \\
\hline $1^{2 J+1} P_{J}, ?^{?}$ & $\Sigma_{b}(6097)^{+} ?$ & $6095.8(2)$ & $\ldots$ & $\Xi_{b}^{\prime}(6227) ?$ & $6226.9(2.1)$ & $\ldots$ \\
\hline
\end{tabular}

$$
(q q)_{d}= \begin{cases}{[u d],} & I=0=\text { spin, in } \Lambda_{Q}, \\ \{u u, u d, d d\}, & I=1=\text { spin, in } \Sigma_{Q} .\end{cases}
$$

while in those with strangeness $=-1$, it can be either of scalar $(\operatorname{spin}=0)$ or axial vector $(\operatorname{spin}=1)$

$$
(q s)_{d}= \begin{cases}{[u s, d s],} & I=1 / 2, \text { spin }=0, \text { in } \Xi_{Q}, \\ \{u s, d s\}, & I=1 / 2, \text { spin }=1, \text { in } \Xi_{Q}^{\prime} .\end{cases}
$$

To confront Eq. (2) with the baryon mass data, we list in Table I the observed masses of the heavy baryons $\Lambda_{Q}$ and the strange heavy baryons $\Xi_{Q}(Q=c, b)$, and in Table II that of the heavy baryons $\Sigma_{Q}$ and the strange heavy baryons $\Xi_{Q}^{\prime}(Q=c, b)$. In Table III, the observed spin-averaged masses for the family of orbitally excited $\mathrm{SH}$ baryons are collected with the weight $2 J+1$ (with $J$ the angular momentum of the baryon), and are fitted by the relation (2), in which the estimated mass can be rewritten as, upon using Eq. (3),

$$
M_{L}=M_{Q}+\sqrt{\pi a L+\left[m_{d}+M_{Q}-m_{\mathrm{bare} Q}^{2} / M_{Q}\right]^{2}} .
$$

In Tables IV, $\mathrm{V}$ and $\mathrm{VI}$, the trajectory parameters reproducing the observed masses in Table I and Table II are listed for the charmed baryons $\Lambda_{c}$ and $\Xi_{c}$, and for the baryons $\Sigma_{c}$ and charm-strange baryons $\Xi_{c}^{\prime}$, respectively, including the ensuing $P$-wave spin-averaged masses predicted by Eq. (6). The resulted parameters are compared with the quark model predictions [17] in the three tables. In Table III, the observed spin-averaged masses are collected for all relevant baryons except for the baryons $\Sigma_{Q}$ and $\Xi_{Q}^{\prime}$ $(Q=c, b)$, and the spin-averaged masses predicted by Eq. (2) via the parameters given in Tables IV, V, and VI are also shown for comparisons. Here, we assumed the orbital

TABLE III. The measured spin-averaged masses of the SH baryons and that predicted by the linear Regge relation (2), with the parameters in Tables IV-VI.

\begin{tabular}{cccccc}
\hline \hline Baryon & $\operatorname{Exp}(\bar{M})$ & This work $(\bar{M})$ & Baryon & $\operatorname{Exp}(\bar{M})$ & This work $(\bar{M})$ \\
\hline$\Lambda_{c}, 1 S$ & 2286.0 & 2286.0 & $\Xi_{c}, 1 S$ & 2467.87 & 2469.0 \\
$\Lambda_{c}, 1 P$ & 2616 & 2618.5 & $\Xi_{c}, 1 P$ & 2808 & 2803.9 \\
$\Lambda_{c}, 1 D$ & 2871 & 2871.1 & $\Xi_{c}, 1 D$ & 3069 & 3071.3 \\
$\Lambda_{b}, 1 S$ & 5619 & 5619 & $\Xi_{b}, 1 S$ & 5792 & 5798.1 \\
$\Lambda_{b}, 1 P$ & 5917 & 5616.3 & $\Xi_{b}, 1 P$ & $\ldots$ & 6125.1 \\
$\Lambda_{b}, 1 D$ & 6148 & 6149.3 & $\Xi_{b}, 1 D$ & $\ldots$ & 6380.9 \\
$\Sigma_{c}, 1 S$ & 2497.0 & 2496.1 & $\Xi_{c}^{\prime}, 1 S$ & 2624 & 2623.1 \\
$\Sigma_{c}, 1 P$ & $\ldots$ & 2774.1 & $\Xi_{c}^{\prime}, 1 P$ & $\ldots$ & 2923.0 \\
$\Sigma_{b}, 1 S$ & 5825 & 5825.0 & $\Xi_{b}^{\prime}, 1 S$ & 5949 & 5949.0 \\
$\Sigma_{b}, 1 P$ & $\cdots$ & 6088.4 & $\Xi_{b}^{\prime}, 1 P$ & $\ldots$ & 6248.2 \\
\hline \hline
\end{tabular}


TABLE IV. The effective masses $(\mathrm{GeV})$ of the charm quark and scalar-diquarks, and the tension $\left(\mathrm{GeV}^{2}\right)$ that match the measured spinaveraged masses of the $\Lambda_{c}$ and the $\Xi_{c}$ in Table I. Here, the RMS error $\chi_{\mathrm{RMS}}=0.001 \mathrm{GeV}$ and $n=u / d$. The comparison with that by quark model is given.

\begin{tabular}{lcccccc}
\hline \hline Parameters & $M_{c}$ & $m_{d}([n n])$ & $a\left(\Lambda_{c}\right)$ & $m_{d}([n s])$ & $a\left(\Xi_{c}\right)$ & $\bar{M}\left(\Lambda_{c}\right)$ \\
\hline This work & 1.44 & 0.535 & 0.212 & 0.718 & 0.255 & 2.618 \\
EFG[17] & 1.55 & 0.710 & 0.18 & 0.948 & 0.18 & 2.617 \\
\hline \hline
\end{tabular}

TABLE V. The effective masses $(\mathrm{GeV})$ of the charm quark and vector-diquarks, and the tension $\left(\mathrm{GeV}^{2}\right)$ that match the measured spinaveraged masses of the $\Sigma_{c}$ and the $\Xi_{c}^{\prime}$ in Table II. Here, the RMS error $\chi_{\mathrm{RMS}}=0.001 \mathrm{GeV}$ and $n=u / d$. The comparison with that by quark model is given.

\begin{tabular}{lcclcccr}
\hline \hline Parameters & $M_{c}$ & $m_{d}(\{n n\})$ & $a\left(\Sigma_{c}\right)$ & $m_{d}(\{n s\})$ & $a\left(\Xi_{c}^{\prime}\right)$ & $\bar{M}\left(\Sigma_{c}\right)$ & $\bar{M}\left(\Xi_{c}^{\prime}\right)$ \\
\hline This work & 1.44 [input] & 0.745 & 0.212 & 0.872 & 0.255 & 2.774 & 2.923 \\
EFG[17] & 1.55 & 0.99 & 0.18 & 1.069 & 0.18 & 2.780 & 2.919 \\
\hline \hline
\end{tabular}

trajectories of the $\Sigma_{c}\left(\Xi_{c}\right)$ and the $\Lambda_{c}\left(\Xi_{c}^{\prime}\right)$ to be parallel, leading to the same string tensions between them.

We also apply Eq. (2), with $m_{d}$ everywhere replaced by the effective masses of light(strange or $u / d$ ) antiquarks, to the heavy-light $(Q \bar{q})$ mesons whose observed masses are listed in Tables VII and VIII, and list in Table IX the determined trajectory parameters, namely, the effective masses $m_{n, s}$ of light $u / d$ quark and the strange quark, and the string tensions $a$, for the $D / D_{s}$ mesons and the $B / B_{s}$ mesons. The resulted parameters are compared to that in the quark model [34] for the heavy-light mesons.

From Table IV one sees that the predicted spin-averaged masses $2616 \mathrm{MeV}$ and $2804 \mathrm{MeV}$ of the $1 P$-wave $\Lambda_{c}$ and $\Xi_{c}$ are very close to the corresponding values $2616 \mathrm{MeV}$ and $2808 \mathrm{MeV}$ in Tables I, respectively. Furthermore, the following remarks are in order.

TABLE VI. The masses $(\mathrm{GeV})$ of the bottom quark and diquarks, and the tension $\left(\mathrm{GeV}^{2}\right)$ that match the measured spin-averaged masses of the $\Lambda_{b}$ and the $\Xi_{b}$ in Table I and the $\Sigma_{b}$ and the $\Xi_{b}^{\prime}$ in Table II. Here, the RMS error $\chi_{\text {RMS }}=0.001$ GeV. The comparison with that by quark model is given.

\begin{tabular}{lccccccr}
\hline \hline Parameters & $M_{b}$ & $m_{d}([n n])$ & $a\left(\Lambda_{b}\right)$ & $m_{d}([n s])$ & $a\left(\Xi_{b}\right)$ & $\bar{M}\left(\Lambda_{b}\right)$ & $\bar{M}\left(\Xi_{b}\right)$ \\
\hline This work & 4.48 & 0.534 & 0.246 & 0.718 & 0.307 & 5.917 & 6.125 \\
EFG [17] & 4.88 & 0.710 & 0.18 & 0.948 & 0.18 & 5.938 & 6.127 \\
\hline \hline Parameters & $M_{b}$ & $m_{d}(\{n n\})$ & $a\left(\Sigma_{b}\right)$ & $m_{d}(\{n s\})$ & $a\left(\Xi_{b}^{\prime}\right)$ & $\bar{M}\left(\Sigma_{b}\right)$ & $\bar{M}\left(\Xi_{b}^{\prime}\right)$ \\
\hline This work & $4.48[$ input] & 0.745 & 0.246 & 0.869 & 0.307 & 6.088 & 6.248 \\
EFG [17] & 4.88 & 0.909 & 0.18 & 1.069 & 0.18 & 6.090 & 6.228 \\
\hline \hline
\end{tabular}

TABLE VII. The observed masses (in MeV) of the charmed and charmed strange mesons [2]. The some of quantum numbers indicated by question marks are quark model predictions, which has not been established experimentally. The errors less than $5 \mathrm{MeV}$ are not indicated.

\begin{tabular}{lcccccc}
\hline \hline State $J^{P}$ & Meson & Mass & EFG [34] & Meson & Mass & EFG [34] \\
\hline $1^{1} S_{0} 0^{-}$ & $D^{ \pm}$ & 1869.7 & 1871 & $D_{s}$ & 1968.3 & 1969 \\
$1^{3} S_{1} 1^{-}$ & $D^{*}(2010)^{ \pm}$ & 2010.3 & 2010 & $D_{s}^{*}\left[J^{P} ?^{?}\right]$ & 2112.2 & 2111 \\
$1^{3} P_{0} 0^{+}$ & $D_{0}^{*}(2400)^{ \pm}$ & $2351(7)$ & 2406 & $D_{s 0}^{*}(2317)$ & 2317.7 & 2509 \\
$1 P_{1} 1^{+}$ & $D_{1}(2430)^{0}$ & $2427(40)$ & 2469 & $D_{s 1}(2460)$ & 2459.5 & 2574 \\
$1 P_{1} 1^{+}$ & $D_{1}(2420)^{ \pm}\left[J^{P} ?^{?}\right]$ & 2423.2 & 2426 & $D_{s 1}(2536)$ & 2535.1 & 2536 \\
$1^{3} P_{2} 2^{+}$ & $D_{2}^{*}(2460)^{ \pm}$ & 2465.4 & 2460 & $D_{s 2}^{*}(2573)$ & 2569.1 & 2571 \\
$1^{3} D_{1} 1^{-}$ & & & 2788 & $D_{s 1}^{*}(2860)$ & $2859(27)$ & 2913 \\
$1 D_{2} 2^{-}$ & & 2850 & & & 2961 \\
$1 D_{2} 2^{-}$ & $D(2740)^{0}\left[J^{P} ?^{?}\right]$ & $2737(12)$ & 2806 & & & 2931 \\
$1^{3} D_{3} 3^{-}$ & $D_{3}^{*}(2750)$ & 2763.5 & 2863 & $D_{s 3}^{*}(2860)$ & $2860(7)$ & 2971 \\
\hline \hline
\end{tabular}


TABLE VIII. The observed masses (in MeV) of the bottomed and bottomed strange mesons [2]. The some of quantum numbers as shown are quark model predictions. The errors less than $5 \mathrm{MeV}$ are not indicated.

\begin{tabular}{lcccccc}
\hline \hline State $J^{P}$ & Meson & Mass & EFG [34] & Meson & Mass & EFG [34] \\
\hline $1^{1} S_{0} 0^{-}$ & $B^{0}$ & 5279.6 & 5280 & $B_{s}$ & 5366.9 & 5372 \\
$1^{3} S_{1} 1^{-}$ & $B^{*}$ & 5324.7 & 5326 & $B_{s}^{*}$ & 5415.4 & 5414 \\
$1^{3} P_{0} 0^{+}$ & $B_{J}^{*}(5732)\left[J^{P} ?^{?}\right]$ & $5698(8)$ & 5749 & & & 5833 \\
$1 P_{1} 1^{+}$ & & & 5774 & $B_{s J}^{*}(5850)\left[J^{P} ?^{?}\right]$ & $5853(15)$ & 5865 \\
$1 P_{1} 1^{+}$ & $B_{1}(5721)^{0}$ & 5726.0 & 5723 & $B_{s 1}(5830)$ & 5828.6 & 5831 \\
$1^{3} P_{2} 2^{+}$ & $B_{2}^{*}(5747)^{0}$ & 5739.5 & 5741 & $B_{s 2}^{*}(5840)$ & 5839.9 & 5842 \\
\hline \hline
\end{tabular}

TABLE IX. The effective masses (in GeV) of quarks that match the observed spin-averaged masses in Table VII and VIII, with $a$ in $\mathrm{GeV}$ and the RMS error $\chi_{\mathrm{RMS}}=0.001 \mathrm{GeV}$. The comparison with that by quark model is given.

\begin{tabular}{lcccccccc}
\hline \hline Parameters & $M_{c}$ & $M_{b}$ & $m_{n}$ & $m_{s}$ & $a(c \bar{n})$ & $a(c \bar{s})$ & $a(b \bar{n})$ & $a(b \bar{s})$ \\
\hline This work & 1.44 [input] & 4.48 [input] & 0.23 & 0.328 & 0.223 & 0.249 & 0.275 & 0.313 \\
EFG [34] & 1.55 & 4.88 & 0.33 & 0.5 & $0.64 / 0.58$ & $0.68 / 0.64$ & $1.25 / 1.21$ & $1.28 / 1.23$ \\
\hline \hline
\end{tabular}

(1) The mass differences between the S-wave $\mathrm{SH}$ baryons $\Sigma_{c}$ and $\Lambda_{c}$ are equal to that between the diquarks (of scalar or vector type) contained in them,

$$
m_{d}\left(\Sigma_{c}\right)-m_{d}\left(\Lambda_{c}\right)=210 \mathrm{MeV}=\bar{M}\left(\Sigma_{c}\right)-M\left(\Lambda_{c}\right)
$$

$m_{d}\left(\Xi_{c}^{\prime}\right)-m_{d}\left(\Xi_{c}\right)=154 \mathrm{MeV}=\bar{M}\left(\Xi_{c}^{\prime}\right)-M\left(\Xi_{c}\right)$.

This imposes a constraint on the masses of the vector diquark which are crucial for predicting, through Eq. (2), the spin-averaged masses of the $P$-wave excitations of the baryons $\Sigma_{c}$ and $\Xi_{c}^{\prime}$. The latter are less established experimentally, as shown in Table II.

(2) The mass values of the heavy quark and light diquarks in our approach are in consistent with that in the relativistic quark model. The string tension $a$ in our approach is flavor-dependent weakly, with a rising about $0.04 \mathrm{GeV}^{2} \quad\left(0.06 \mathrm{GeV}^{2}\right)$ for the charmed(bottom) baryons when diquark flavor in them changes from $n n$ to $n s(n=u, d)$.

(3) Once determined, the diquark masses of both types can be applied to explore the Regge behaviors in the bottom partner of the charmed baryons, for which the less data are available experimentally.

\section{INCLUDING MASS SPLITTING FOR ORBITAL EXCITATIONS}

To estimate the mass splitting in orbitally excited charmed and bottom baryons $Q q q(Q=c, b, q=u / d$, s, $q q \neq s s$ ), one needs to consider the spin-dependent interaction which was ignored in Eq. (2). In the light of QCD string, the orbital motion of QCD string [34] contributes dominantly to total angular moment $L$ of the system, which makes the quark-diquark potential nontrivial ( $L$ dependent) effectively. For simplicity, we follow Ref. [35] to compute the spin-dependent mass for $L=0,1$ and $2\left(\Lambda_{Q}, \Xi_{Q}\right.$ case only $)$ via the scaling relation between heavy baryons $Q q q$ and their meson partners $Q \bar{q}$ (note that the color-structure similarity between baryon and meson is implicitly assumed in the quark-diquark picture). The spin-dependent interaction we consider is $[17,35]$

$$
\begin{gathered}
H^{S D}=a_{1} \mathbf{L} \cdot \mathbf{S}_{d}+a_{2} \mathbf{L} \cdot \mathbf{S}_{Q}+b \mathbf{S}_{12}+c \mathbf{S}_{d} \cdot \mathbf{S}_{Q}, \\
\mathbf{S}_{12}=3 \mathbf{S}_{d} \cdot \hat{\mathbf{r}} S_{Q} \cdot \hat{\mathbf{r}}-\mathbf{S}_{d} \cdot \mathbf{S}_{Q},
\end{gathered}
$$

where the first two terms are spin-orbit energies, the third is the tensor energy, and the last describes the hyperfine splitting between the heavy quark with spin $\mathbf{S}_{Q}$ and diquark with spin $\mathbf{S}_{d}$. Here, $\mathbf{L}$ is the orbital angular momentum of the baryons. The first term ( $a_{1}$-term) is obviously larger than the others (especially for the bottom systems) as the later all involve the magnetic moment $\mathbf{S}_{Q} / M_{Q}$ of heavy quark when viewing Eq. (9) as a spin-relevant relativistic correction.

(A) The $S$-wave baryons $(L=0)$. In this case, only the last term survives in Eq. (9), $H^{S D}=c \mathbf{S}_{d} \cdot \mathbf{S}_{Q}$. Note that $\left\langle\mathbf{S}_{d} \cdot \mathbf{S}_{Q}\right\rangle=\left[S(S+1)-S_{Q}\left(S_{Q}+1\right)-S_{d}\left(S_{d}+1\right)\right] / 2$ becomes 0 when the conserved total quark spin $S(=J$ here $)=1 / 2$ and $S_{d}=0$, and has the value -1 and $1 / 2$, when $S_{d}=1$ and $S=1 / 2,3 / 2$, respectively. Thus, the mass for the $1 S$-wave states is

$$
M(1 S)_{S_{d}=0}=\bar{M}_{L=0},\left(\Lambda_{Q} / \Xi_{Q}\right)
$$




$$
M(1 S)_{S_{d}=1}=\bar{M}_{L=0}+c\left[\begin{array}{rr}
-1 & 0 \\
0 & 1 / 2
\end{array}\right], \quad\left(\Sigma_{Q} / \Xi_{Q}^{\prime}\right) .
$$

For the baryon $\Lambda_{Q}$ and $\Xi_{Q}$ containing an light scalar diquark(spin $S_{d}=0$ ), the total spin $S=1 / 2=J$. This corresponds to a single baryon ground state $1^{2} S_{1 / 2}$. The ground state masses of the baryons $\Lambda_{Q}$ and $\Xi_{Q}$ are given simply by their spin-independent masses in Eq. (2). To estimate $c$ for the baryon $\Sigma_{Q}$ and $\Xi_{Q}^{\prime}$ which contain the light vector diquark (spin $S_{d}=1$ ), we employ the following scaling relation to the partner in heavy mesons,

$$
c(Q q q)=\frac{m_{q}}{m_{d}(q q)} c(Q \bar{q})
$$

which stems from the inverse-mass scaling inherent in the contact interaction $(\sim$ the diquark magnetic moment $\left.\mathbf{S}_{d} / m_{d}\right)$. Extracting $c(Q \bar{q})$ from the splitting between the $Q \bar{q}$ meson mass $M\left(1^{3} S_{1}, 1^{-}\right)$and $M\left(1^{1} S_{0}, 0^{-}\right)$in the case of the $D$ mesons (see Table VII):

$c(Q \bar{q})=M\left(1^{3} S_{1}, 1^{-}\right)-M\left(1^{1} S_{0}, 0^{-}\right)=140.6 \mathrm{MeV}$,

one estimates, by Eq. (12),

$$
c=140.6 \times\left\{\begin{array}{l}
230 / 745 \\
230 / 872
\end{array}\right\}=\left\{\begin{array}{l}
43.4\left(\Sigma_{c}\right) \\
37.1\left(\Xi_{c}^{\prime}\right)
\end{array}\right\},
$$

Having $c$ given by Eps. (14), Eqs. (10)-(11) give masses for the S-wave charmed baryons, as listed in Table I and Table II, respectively.

(B) The $P$-wave baryons $(L=1)$. For the baryon $\Lambda_{Q}$ and $\Xi_{Q}$ containing spin $=0$ diquarks, the $P$-wave states correspond to two baryon states of $J^{P}=1 / 2^{-}$and $3 / 2^{-}$. This can be written as $\left[0_{d} \otimes\left(\frac{1}{2}\right)_{Q}\right]_{S} \otimes 1_{L}=\frac{1}{2} \oplus \frac{3}{2}$ in the notation of symmetry group, where the notations $\otimes$ and $\oplus$ stand for the coupling(adding of the angular momentum in the standard terminology) and the juxtaposition of the angular momentum shown. In the charm sectors, for instance, there are four $P$-wave candidates $\Lambda_{c}(2595)^{+}$and $\Lambda_{c}(2625)^{+}$, $\Xi_{c}(2790)^{+}$and $\Xi_{c}(2815)^{+}$[2] (see Table I). The masses and possible quantum numbers for the observed low-lying baryons $\Lambda_{c}$ and $\Xi_{c}$ are summarized in Table I.

For the baryon $\Sigma_{Q}$ and $\Xi_{Q}^{\prime}$ containing spin $=1$ diquarks, the five $P$-wave states $J=1 / 2^{\prime}, 3 / 2^{\prime}, 1 / 2,3 / 2$, and $5 / 2$ with negative parity are allowed:

$$
\begin{aligned}
{\left[1_{d} \otimes\left(\frac{1}{2}\right)_{Q}\right]_{S} \otimes 1_{L} } & =\left[\frac{1^{\prime}}{2} \oplus \frac{3}{2}\right]_{S} \otimes 1_{L}, \\
& =\frac{1}{2} \oplus \frac{3}{2} \oplus \frac{1^{\prime}}{2} \oplus \frac{3^{\prime}}{2} \oplus \frac{5}{2} .
\end{aligned}
$$

The current candidates of these $P$-wave charmed baryons are the $\Sigma_{c}(2800)^{0,+,++}$ in the nonstrange sector and the $\Xi_{c}(2930)^{0,+}$ in the strange sector, whose spin and parity have not been established yet [2]. The quark model assignments are given by Eq. (1). The masses and possible quantum numbers for the observed low-lying baryons $\Sigma_{c}$ and $\Xi_{c}^{\prime}$ are summarized in Table II, with the question marks indicating that their $J^{P}$ assignments are unknown experimentally.

A normal scheme (the $L-S$ coupling) of computing mass splitting by Eq. (9) is to couple the diquark spin $\mathbf{S}_{d}$ to the heavy quark spin $\mathbf{S}_{Q}$ first and then to the orbital angular momentum $\mathbf{L}$ of the system, as shown in (15) for $S_{d}=1$ case. In the $L-S$ basis the two $J=1 / 2$ states and the two $J=3 / 2$ states are usually mixed unless $a_{1}=a_{2}$. They are eigenstates of $2 \times 2$ matrices $M_{J}$ given in the basis ${ }^{2} P_{J},{ }^{4} P_{J}$. Using the $1 P$-wave wavefunctions of the heavy quark-diquark systems in the basis of $\left|L_{z}, S_{Q z}, S_{d z}\right\rangle$, these matrices can be evaluated and given by (Appendix B)

$$
\begin{aligned}
& \left\langle\mathbf{L} \cdot \mathbf{S}_{d}\right\rangle_{J=\frac{1}{2}}=\left[\begin{array}{rr}
-\frac{4}{3} & -\frac{\sqrt{2}}{3} \\
-\frac{\sqrt{2}}{3} & -\frac{5}{3}
\end{array}\right] \\
& \left\langle\mathbf{L} \cdot \mathbf{S}_{d}\right\rangle_{J=\frac{3}{2}}=\left[\begin{array}{rr}
\frac{2}{3} & -\frac{\sqrt{5}}{3} \\
-\frac{\sqrt{5}}{3} & -\frac{2}{3}
\end{array}\right]
\end{aligned}
$$

and $\left\langle\mathbf{L} \cdot \mathbf{S}_{d}\right\rangle_{J=5 / 2}=1, \quad\left\langle\mathbf{L} \cdot \mathbf{S}_{Q}\right\rangle_{J=5 / 2}=1 / 2$. The two matrices above have the eigenvalues $-1,-2$ for $J=1 / 2$ and the eigenvalues \pm 1 for $J=3 / 2$.

One can also choose an alternative scheme(the $j$-j coupling) in which the diquark spin $\mathbf{S}_{d}$ couples to $\mathbf{L}$ first and then to the conserved spin $\mathbf{S}_{Q}$ of the heavy quark. In the $P$-wave case $(L=1)$, for instance, this scheme reads,

$$
\left[0_{d} \otimes 1_{L}\right]_{j} \otimes\left(\frac{1}{2}\right)_{Q}=1_{j} \otimes\left(\frac{1}{2}\right)_{Q}=\frac{1}{2} \oplus \frac{3}{2},
$$

for the baryons $\Lambda_{Q}$ and $\Xi_{Q}$, and takes form

$$
\begin{aligned}
{\left[1_{d} \otimes 1_{L}\right]_{j} \otimes\left(\frac{1}{2}\right)_{Q} } & =\{0,1,2\}_{j} \otimes\left(\frac{1}{2}\right)_{Q} \\
& =\frac{1}{2} \oplus \frac{1^{\prime}}{2} \oplus \frac{3}{2} \oplus \frac{3^{\prime}}{2} \oplus \frac{5}{2},
\end{aligned}
$$

for the baryons $\Sigma_{Q}$ and $\Xi_{Q}^{\prime}$. Since the interactions other than the $\mathbf{L} \cdot \mathbf{S}_{d}$ term in (9) are suppressed by $1 / M_{Q}$, one can compute the mass splitting in a basis in which $\mathbf{L} \cdot \mathbf{S}_{d}$ is diagonal, with the other interactions treated perturbatively.

Notice that $\left\langle\mathbf{L} \cdot \mathbf{S}_{d}\right\rangle=\left[j(j+1)-L(L+1)-S_{d}\left(S_{d}+1\right)\right] / 2$ takes values $-2,-1$ and 1 when $j=0,1,2$, respectively, one can use the eigenfunction of the $\mathbf{L} \cdot \mathbf{S}_{d}$ to evaluate the mass splitting operators in Eq. (9) in the $j$-j coupling 
TABLE X. The matrix elements of the mass splitting operators in the $P$-wave baryon states in the $j$ - $j$ coupling.

\begin{tabular}{lccc}
\hline \hline$(J, j)$ & $\left\langle\mathbf{L} \cdot \mathbf{S}_{Q}\right\rangle$ & $\left\langle\mathbf{S}_{12}\right\rangle$ & $\left\langle\mathbf{S}_{d} \cdot \mathbf{S}_{Q}\right\rangle$ \\
\hline$(1 / 2,0)$ & 0 & 0 & 0 \\
$(1 / 2,1)$ & $-\frac{1}{2}$ & -1 & $-\frac{1}{2}$ \\
$(3 / 2,1)$ & $\frac{1}{4}$ & $\frac{1}{2}$ & $\frac{1}{4}$ \\
$(3 / 2,2)$ & $-\frac{3}{4}$ & $\frac{3}{10}$ & $-\frac{3}{4}$ \\
$(5 / 2,2)$ & $\frac{1}{2}$ & $-\frac{1}{5}$ & $\frac{1}{2}$ \\
\hline \hline
\end{tabular}

(Appendix B). The results are listed in Table X, from which the mass splitting $M(J, j)=\left\langle J, j\left|H^{S D}\right| J, j\right\rangle$ can be evaluated in the $|J, j\rangle$ basis. Written in terms of three parameters $\left(a_{1}, a_{2}+c, b\right)$, the results are (see Ref. [36] for details, which corrected an error in Ref. [35])

$$
\begin{gathered}
M^{S D}(1 / 2,0)=-2 a_{1}, \\
M^{S D}(1 / 2,1)=-a_{1}-\frac{1}{2}\left(a_{2}+c\right)-b, \\
M^{S D}(3 / 2,1)=-a_{1}+\frac{1}{4}\left(a_{2}+c\right)+\frac{1}{2} b, \\
M^{S D}(3 / 2,2)=a_{1}-\frac{3}{4}\left(a_{2}+c\right)+\frac{3}{10} b, \\
M^{S D}(5 / 2,2)=a_{1}+\frac{1}{2}\left(a_{2}+c\right)-\frac{1}{5} b .
\end{gathered}
$$

One additional constraint stems from the vanishing of these splitting as a sum: $\sum(2 J+1) M^{S D}(J, j)=0$, which is

$$
\begin{aligned}
& 10 M^{S D}(1 / 2,0)-15 M^{S D}(1 / 2,1)+8 M^{S D}(3 / 2,2) \\
& \quad=3 M^{S D}(5 / 2,2) .
\end{aligned}
$$

Following Ref. [35], one can calculate the parameters $\left\{a_{1}, a_{2}, b\right\}$ in Eq. (9) for the heavy baryon $Q q q(q=$ $u / d, s)$ by assuming the following mass scaling, which relates these parameters to that for the heavy mesons $Q \bar{q}$ with effective antiquark mass $m_{q}$, or relates the parameters among the heavy flavor partners ( $b q q$ and $c q q)$,

$$
\begin{gathered}
a_{1}(Q q q)=a_{1}(Q \bar{q})\left(\frac{m_{q}}{m_{d}(q q)}\right), \\
a_{1}(b)=a_{1}(c), \quad a_{2}(b)=a_{2}(c)\left(\frac{M_{c}}{M_{b}}\right), \\
b(b)=b(c)\left(\frac{M_{c}}{M_{b}}\right),
\end{gathered}
$$

where $b, c$ in parenthesis stand for the bottom and charm sectors. In evaluating the coupling $a_{2}$ for the charmed baryons, a simple way is to estimate it via that for the charmed mesons by resembling the charmed baryon $c q q$ to the charmed meson $c \bar{q}$ systems. Considering the finite mass effects of the charm quark which differ between the charmed baryons and the charmed mesons, we use a recoil factor $\left(1+m_{d} / M_{Q}\right)$ to partially remedy the uncertainty that may happen in this resembling,

$$
a_{2}(c q q)=\frac{a_{2}(c \bar{q})}{1+m_{d} / M_{c}} .
$$

In the following, we describe very briefly how to evaluate the parameters $\left\{a_{1}, a_{2}, b\right\}$ for the charmed-strange mesons for which the spin-dependent Hamiltonian is given by Eq. (9) in which $\mathbf{S}_{d}$ is replaced by $\mathbf{S}_{q}$. First, one can derive a counterpart of Eqs. (16)-(20) for $Q \bar{q}$ system via a calculation similar to the $P$-wave heavy baryons and obtain the mass for the $P$-wave spin multiplets of the heavy mesons $(Q \bar{q})$ in the $|J, j\rangle$ states $\left(j=L_{3}+S_{3}, L=1\right)$. The results are [Appendix C: Eq. $(\mathrm{C} 8)-(\mathrm{C} 11)]$ :

$$
\begin{gathered}
M(0,1 / 2)=\bar{M}-a_{1}-a_{2}-b-\frac{c}{4}, \\
M(1,1 / 2)=\bar{M}-a_{1}+\frac{1}{3} a_{2}+\frac{1}{3} b-\frac{c}{12}, \\
M(1,3 / 2)=\bar{M}+\frac{1}{2} a_{1}-\frac{5}{6} a_{2}+\frac{1}{6} b-\frac{5 c}{12}, \\
M(2,3 / 2)=\bar{M}+\frac{1}{2} a_{1}+\frac{1}{2} a_{2}-\frac{1}{10} b+\frac{c}{4} .
\end{gathered}
$$

Second, confronting the observed masses of the $D_{s}$ mesons in the $P$-wave (see Table VII), one can find the parameters $\left\{a_{1}, a_{2}, b\right\}$ for the heavy mesons $D_{s}$ reproducing their observed mass spectrum via Eqs. (16)-(20). Ignoring $c$ (vanishes almost in the $P$-wave), one can find lastly [35] [see Appendix C: Eq. (C12)]:

$\left\{\begin{array}{llll}\bar{M}(\mathrm{MeV}), & a_{1}(\mathrm{MeV}), & a_{2}(\mathrm{MeV}) & b(\mathrm{MeV}) \\ 2513.4, & 89.4, & 40.7, & 65.6 .\end{array}\right\}$

Given $a_{1}$ in Eq. (29) for the $D_{s}$ mesons in $P$-wave, the diquark masses in Table IV and $\mathrm{V}$ and the strange quark mass in Table IX, Eq. (22) gives

$$
\begin{aligned}
\Sigma_{c}, 1 P: \quad a_{1} & =a_{1}(c \bar{s})\left(\frac{m_{s}}{m_{d}(\{q q\})}\right)=89.4 \mathrm{MeV}\left(\frac{328}{745}\right) \\
& =39.4 \mathrm{MeV}, \\
\Xi_{c}^{\prime}, 1 P: \quad a_{1} & =a_{1}(c \bar{s})\left(\frac{m_{s}}{m_{d}(\{q s\})}\right)=89.4 \mathrm{MeV}\left(\frac{328}{872}\right) \\
& =33.6 \mathrm{MeV} .
\end{aligned}
$$

For the charmed baryons $\Sigma_{c}$ and $\Xi_{c}^{\prime}$, Eq. (24) with $m_{d}$ given in Table $\mathrm{V}$ gives 
$a_{2}\left(\Sigma_{c}, 1 P\right)=\frac{a_{2}\left(D_{s}\right)}{1+m_{d} / M_{c}}=\frac{40.7}{1.517}=26.8 \mathrm{MeV}$,

$a_{2}\left(\Xi_{c}^{\prime}, 1 P\right)=\frac{a_{2}\left(D_{s}\right)}{1+m_{d} / M_{c}}=\frac{40.7}{1.606}=25.3 \mathrm{MeV}$,

while for the charmed baryons $\Lambda_{c}$ and $\Xi_{c}$, the parameter $a_{2}$ become slightly larger when using Eq. (29) and the diquark masses given in Table IV. We take $a_{2}\left(\Lambda_{c}, 1 P\right) \simeq a_{2}\left(\Sigma_{c}, 1 P\right)$ and $a_{2}\left(\Xi_{c}, 1 P\right) \simeq a_{2}\left(\Xi_{c}^{\prime}, 1 P\right)$ for simplicity as this varying in $a_{2}$ is small at the mass scale of the $P$-wave charmed baryons, as shown explicitly in Figs. 1-2. Equations (31) are in consistent with the values of $a_{2}$ that match the observed $P$-wave masses of the baryons $\Lambda_{c}$ and $\Xi_{c}$ :

$$
a_{2}\left(\Lambda_{c}\right)=24 \mathrm{MeV}, \quad a_{2}\left(\Xi_{c}\right)=16 \mathrm{MeV} .
$$

These two values follows from the mass differences (see Table I) between the $J=3 / 2^{-}$and $J=1 / 2^{-}$states of the baryon $\Lambda_{c}$ and the $\Xi_{c}$, respectively, which depend only on the second term in Eq. (9) when $\mathbf{S}_{d}=0$, that is, $M\left(J=3 / 2^{-}\right)-M\left(J=1 / 2^{-}\right)=3 a_{2} / 2$.

As $\mathbf{S}_{d}=0$ for the $\Lambda_{c}$ and the $\boldsymbol{\Xi}_{c}$, the mass splitting operator for them simplifies, $H^{S D}=a_{2} \mathbf{L} \cdot \mathbf{S}_{Q}$, which gives the mass in the $|J=1 / 2, j=1\rangle$ and $|J=3 / 2, j=1\rangle$ :

$M\left(\Lambda_{c} / \Xi_{c}, 1 P\right)=\bar{M}_{L=1}+a_{2}\left(\Lambda_{c} / \Xi_{c}\right)\left[\begin{array}{rr}-1 & 0 \\ 0 & 1 / 2\end{array}\right]$,

where the expectation values of $\mathbf{L} \cdot \mathbf{S}_{Q}$ is given by $\left[J(J+1)-L(L+1)-S_{Q}\left(S_{Q}+1\right)\right] / 2$, taking values -1 for $J=1 / 2$ and $+1 / 2$ for $J=3 / 2$. The computed masses via Eqs. (33) and (31) for the $P$-wave $\Lambda_{c} / \Xi_{c}$ are collected in Table I.

Putting all together and ignoring the spin-spin $c$-term (quite small) in the $P$-wave, it follows from Eqs. (16)-(20) that the mass splitting in the $P$-wave are

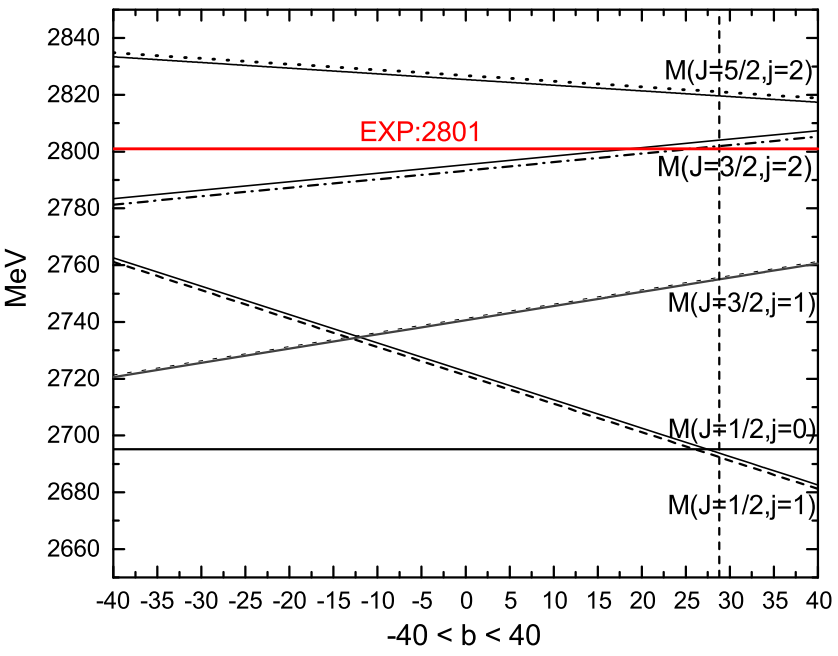

FIG. 1. The masses of the $P$-wave $\Sigma_{c}$ against the parameter $b$.

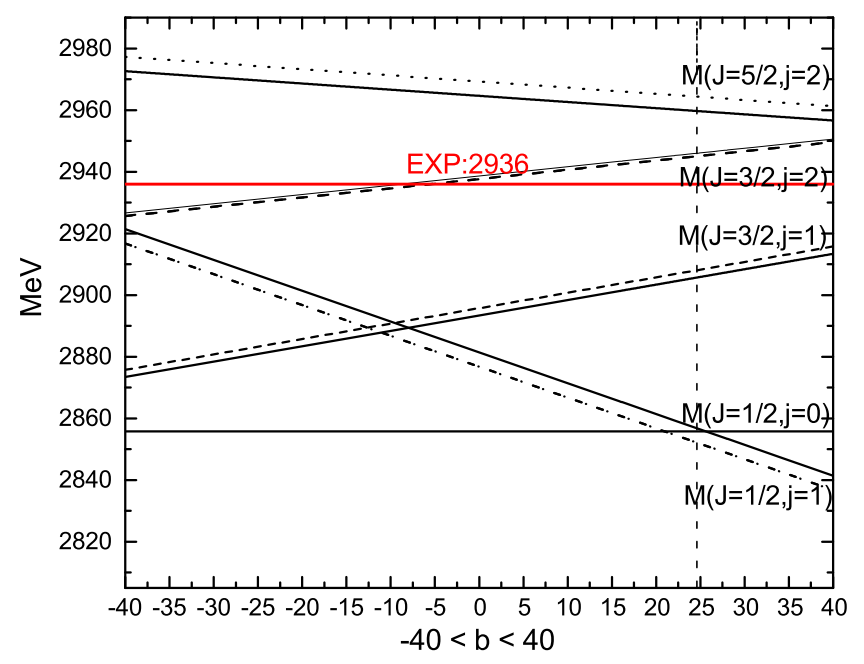

FIG. 2. The masses of the $P$-wave $\Xi_{c}^{\prime}$ against the parameter $b$.

$$
\begin{gathered}
M(1 / 2,0)=2694.74, \\
M(1 / 2,1)=2722.64-b, \\
M(3 / 2,1)=2740.37+\frac{1}{2} b, \\
M(3 / 2,2)=2795.63+\frac{3}{10} b, \\
M(5 / 2,2)=2825.63-\frac{1}{5} b .
\end{gathered}
$$

for the baryon $\Sigma_{c}$, and

$$
\begin{gathered}
M(1 / 2,0)=2855.76, \\
M(1 / 2,1)=2881.38-b, \\
M(3 / 2,1)=2893.38+\frac{1}{2} b, \\
M(3 / 2,2)=2938.62+\frac{3}{10} b, \\
M(5 / 2,2)=2964.62-\frac{1}{5} b,
\end{gathered}
$$

for the baryon $\Xi_{c}^{\prime}$, which are plotted against $b$ in Figs. 1 and 2, respectively. Each pair of the adjacent lines corresponds to the choice $a_{2}$ given in Eq. (31) (dotted lines) and Eq. (32) (solid lines), respectively. As shown in Fig. 1, for moderate values of $b$, there is a clear separation between the three lowest masses $M(1 / 2, j=0), \quad M(1 / 2, j=1)$, $M(3 / 2, j=1)$, and the two highest masses $M(3 / 2, j=2)$ and $M(5 / 2, j=2)$.

Without loss of the generality, we assume that $b$ is of same order with $a_{2}$ (note that $a_{2}=b$ in the quark model [37] of the heavy-light mesons in the heavy quark limit). 
The mass function $M(3 / 2, j=2)$ in Fig. 1 approaches the observed mass of the $\Sigma_{c}(2800)^{0,+,++}$ at $b$ around $20 \mathrm{MeV}$, while the mass function $M(3 / 2, j=2)$ meets the observed averaged mass $2936 \mathrm{MeV}$ of the $\Xi_{c}(2930)$ and $\Xi_{c}(2942)$, Also, the mass function $M(3 / 2, j=1)$ slowly tends to approach the mass of the $\Xi_{c}(2930)$ at larger $b$ in Fig. 2. In both cases, the $P$-wave states with the spin-parity $J^{P}=$ $3 / 2^{-}$is favored. The states with $J^{P}=3 / 2^{-}$and $5 / 2^{-}$can be the states most likely being relatively narrow and thereby easier to observe when they decay via the higher partial wave( $D$-wave), as discussed in Sec. IV.

(C) The $D$-wave baryons $(L=2)$. We consider only the baryon $\Lambda_{Q}$ and $\Xi_{Q}$ containing spin $=0$ diquarks due to the limited experimental information for the charmed mesons $(c \bar{q})$ in $D$-wave on which our calculation depends. Two possible $D$-wave states are the baryon states of $J^{P}=3 / 2^{+}$ and $5 / 2^{+}$, which can be written as

$$
\left[0_{d} \otimes\left(\frac{1}{2}\right)_{Q}\right]_{S} \otimes 2_{L}=\frac{3}{2} \oplus \frac{5}{2} .
$$

In the charm sectors, for instance, there are four $D$-wave candidates, the $\Lambda_{c}(2860)^{+}$, the $\Lambda_{c}(2880)^{+}$, the $\Lambda_{c}(3055)^{+}$ and the $\Xi_{c}(3080)^{+}$[2], as shown in Table I where the other masses and their quantum numbers are also summarized.

Given $\mathbf{S}_{d}=0$ for the baryon $\Lambda_{Q}$ and $\Xi_{Q}$ the mass splitting operator is $H^{S D}=a_{2} \mathbf{L} \cdot \mathbf{S}_{Q}$, which gives the mass in the basis $|3 / 2, j=2\rangle$ and $|5 / 2, j=2\rangle$ :

$$
M\left(\Lambda_{Q} / \Xi_{Q}, 1 D\right)=\bar{M}_{L=2}+a_{2}\left[\begin{array}{rr}
-3 / 2 & 0 \\
0 & 1
\end{array}\right] .
$$

Employing the parametrized mass in Eqs. (C19-C22) stemming from Eq. (9) in the $j-j$ coupling and confronting it with the observed masses of the $D$ mesons in $D$-wave in Table XII (see Appendix C), one finds $a_{2}=12.6 \mathrm{MeV}$ [see Eq. (C23) for details]. It follows from Eq. (24), with $m_{d}$ in Table IV,

$$
a_{2}(c q q)=\frac{12.6 \mathrm{MeV}}{1+m_{d} / 1.44}=\left\{\begin{array}{c}
9.2 \mathrm{MeV}\left(\Lambda_{c}\right) \\
8.4 \operatorname{MeV}\left(\Xi_{c}\right)
\end{array}\right\} .
$$

by which all computed masses of the $D$-wave charmed baryons in Table I follow.

\section{MASS SPLITTING FOR THE SINGLY BOTTOM BARYONS}

One can apply the calculational method similar to Sec. III to the singly bottom baryons. Differing from the charmed baryons, one can improve the computational accuracy for the bottom baryons via the scaling relation to the charmed baryons(note that there are few higher partial waves for the $B_{s}$ mesons in Table VIII). The masses and the quantum numbers for the observed low-lying baryons $\Lambda_{b}$ and $\Xi_{b}$ are summarized in Table I, whereas in Table II, we list mainly the observed lowest baryons $\Sigma_{b}$ and $\Xi_{b}^{\prime}$ and the newly observed excited baryons $\Sigma_{b}(6097)$ and $\Xi_{b}^{\prime}(6227)$, including the resonances $\Lambda_{b}(6146)$ and $\Lambda_{b}(6152)$ [38] discovered very recently by the LHCb Collaboration.

(A) The $S$-wave baryons $(L=0)$. Only the last (contact) term in Eq. (9) survives for the bottom baryons $\left(\Sigma_{b}, \Xi_{b}^{\prime}\right)$ while Eq. (9) vanishes for the bottom baryons $\left(\Lambda_{b}, \Xi_{b}\right)$ for which both $\mathbf{L}$ and $\mathbf{S}_{d}$ vanish. Considering the contact term is suppressed by the magnetic moment $\mathbf{S}_{Q} / M_{Q}$, the computation of the parameter $c$ for the bottom baryons $\left(\Sigma_{b}, \Xi_{b}^{\prime}\right)$ can be obtained by the following heavy-quark mass scaling

$$
c=c\left(\Sigma_{c}\right)\left(\frac{M_{c}}{M_{b}}\right)=\left\{\begin{array}{l}
12.8\left(\Sigma_{b}\right) \\
10.9\left(\Xi_{b}^{\prime}\right)
\end{array}\right\} .
$$

Given $c$ in Eq. (46), Eqs. (10)-(11) lead to the masses for the S-wave bottom baryons, as listed in Tables I and II, respectively.

(B) The $P$-wave baryons $(L=1)$. A classification that is similar to the charmed baryons in Sec. III B can be given to the baryon state (namely, $Q \rightarrow b$ ). Using the scaling (23) for the bottom hadrons where $Q=b$, one can calculate $a_{1}$,

$$
\begin{aligned}
& \Sigma_{b}: a_{1}(1 P)=a_{1}\left(\Sigma_{c}(1 P)\right)=39.4 \mathrm{MeV}, \\
& \Xi_{b}^{\prime}: a_{1}(1 P)=a_{1}\left(\Xi_{c}^{\prime}(1 P)\right)=33.6 \mathrm{MeV} .
\end{aligned}
$$

For the parameter $a_{2}$ for the singly bottom baryons, the scaling (23) gives, using $a_{2}$ for the $\mathrm{SH}$ mesons given in Eq. (32),

$a_{2}\left(\Sigma_{b}\right)=a_{2}\left(\Sigma_{c}\right)\left(\frac{M_{c}}{M_{b}}\right)=24\left(\frac{1.44}{4.48}\right)=7.7 \mathrm{MeV}$,
$a_{2}\left(\Xi_{b}^{\prime}\right)=a_{2}\left(\Xi_{c}^{\prime}\right)\left(\frac{M_{c}}{M_{b}}\right)=16\left(\frac{1.44}{4.48}\right)=5.1 \mathrm{MeV}$.

Note that they are smaller than $a_{1}$ roughly by factor of $1 / 5$. For the $\Lambda_{b} / \Xi_{b}$, Eq. (23) leads to, using $a_{2}$ in the charm sector given in Eq. (31),

$$
\begin{aligned}
& a_{2}\left(\Lambda_{b}\right)=a_{2}\left(\Lambda_{c}\right)\left(\frac{1.44}{4.48}\right)=8.61 \mathrm{MeV} \\
& a_{2}\left(\Xi_{b}\right)=a_{2}\left(\Xi_{c}\right)\left(\frac{1.44}{4.48}\right)=8.13 \mathrm{MeV}
\end{aligned}
$$

Including the spin-dependent mass splitting, the relation similar to Eq. (33) yields 
$M\left(\Lambda_{b} / \Xi_{b}, 1 P\right)=\bar{M}_{L=1}\left(\Lambda_{b} / \Xi_{b}\right)+a_{2}\left(\Lambda_{b} / \Xi_{b}\right)\left[\begin{array}{rr}-1 & 0 \\ 0 & 1 / 2\end{array}\right]$,

which gives, combining with the spin-averaged masses in Table VI and Eq. (48), the mass predictions for the $P$-wave baryons $\Lambda_{b}$ and $\Xi_{b}$. The results as collected in Table I.

Knowing the coupling strengths $a_{1,2}$ and adding the spinindependent masses in Table VI to the mass splitting (16)(20), one can find the masses of the $P$-wave spin multiplets,

$$
\begin{gathered}
M(1 / 2,0)=6009, \\
M(1 / 2,1)=6046-b, \\
M(3 / 2,1)=6050+\frac{1}{2} b, \\
M(3 / 2,2)=6124+\frac{3}{10} b, \\
M(5 / 2,2)=6130-\frac{1}{5} b,
\end{gathered}
$$

for the bottom baryon $\Sigma_{b}^{+}$, and

$$
\begin{gathered}
M(1 / 2,0)=6181 \\
M(1 / 2,1)=6211-b, \\
M(3 / 2,1)=6216+\frac{1}{2} b, \\
M(3 / 2,2)=6277+\frac{3}{10} b, \\
M(5 / 2,2)=6285-\frac{1}{5} b,
\end{gathered}
$$

for the baryon $\Xi_{b}^{\prime}$, which are plotted against $b$ in Figs. 3 and 4 , respectively. Note that two adjacent lines in the plots are very close and not distinguishable for the two choices in Eq. (47) and Eq. (48) and hence only the later case is shown in Fig. 3 and Fig. 4.

In Fig. 3, the observed mass of the $\Sigma_{b}(6097)^{-}$pass through the gap between two mass functions $M(3 / 2, j=$ 1,2) while in Fig. 4 the line of the mass function $M(3 / 2, j=1)$ approaches the mass of the $\Xi_{b}^{\prime}(6227)^{-}$at about $b=20$ assuming $b$ is positive. In former case, agreement with data can be achieved most likely when the states $|3 / 2, j=1,2\rangle$ mixes. In both cases, the spinparity $J^{P}=3 / 2^{-}$is favored for the $\Sigma_{b}(6097)^{-}$and $\Xi_{b}^{\prime}(6227)^{-}$. The states with $J^{P}=3 / 2^{-}$are easier to observe since they decay strongly only via $D$-wave and are relatively narrow.

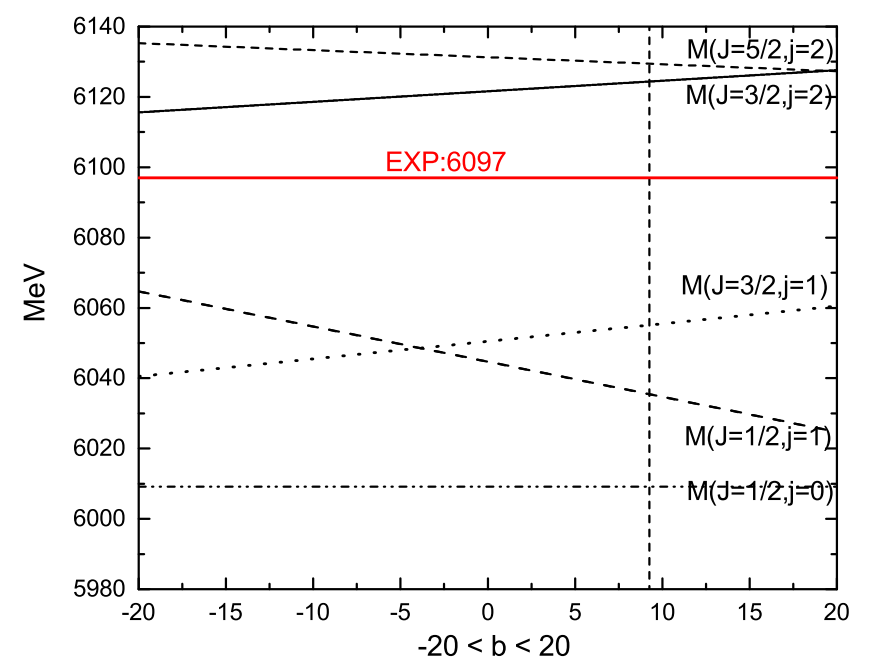

FIG. 3. The masses of the $P$-wave $\Sigma_{b}$ against the parameter $b$.

(C) The $D$-wave baryons $\Lambda_{b} / \Xi_{b}$. We compute, as an example, the masses of the $\Lambda_{b}(1 D)$. Using the trajectory parameters in Table VI, and $M_{b} v_{b}^{2}=4.48\left(1-4.18^{2} / 4.48^{2}\right)=$ $0.5799 \mathrm{GeV}$, Eqs. (2)-(3) yield a spin-averaged mass for the $D$-wave,

$$
\begin{aligned}
\bar{M}(1 D) & =4.48+\sqrt{2 \pi \cdot 0.246+[0.534+0.5799]^{2}} \\
& =6.14927 \mathrm{GeV}
\end{aligned}
$$

which agrees remarkably well with the observed spinaveraged mass $6149.97 \mathrm{MeV}$ of the new resonances $\Lambda_{b}\left(3 / 2^{+}, 5 / 2^{+}\right)$reported very recently by $\mathrm{LHCb}$ [38]:

$$
\begin{aligned}
& M\left[\Lambda_{b}(6146)^{0}\right]=6146.17 \pm 0.33 \pm 0.22 \pm 0.16 \mathrm{MeV} \\
& M\left[\Lambda_{b}(6152)^{0}\right]=6152.51 \pm 0.26 \pm 0.22 \pm 0.16 \mathrm{MeV}
\end{aligned}
$$

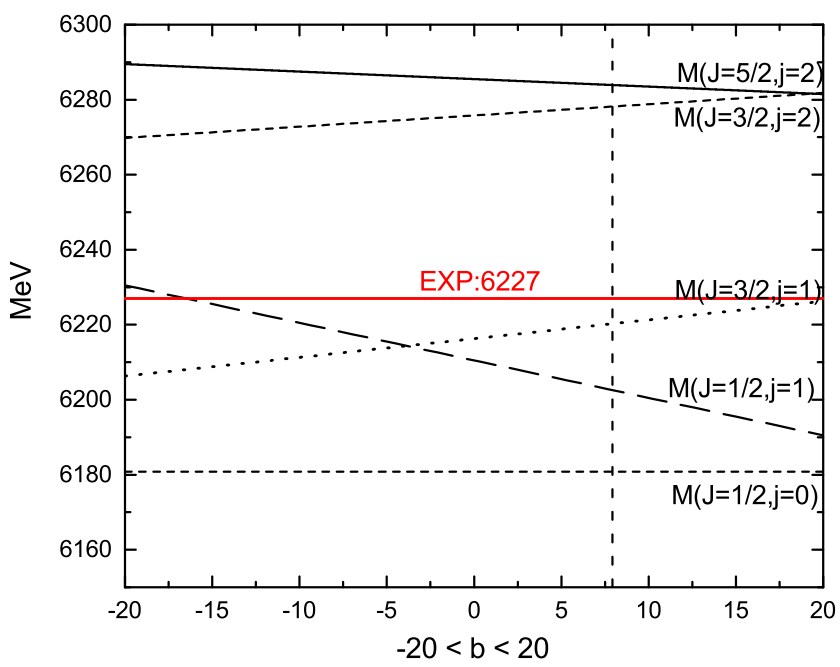

FIG. 4. The masses of the $P$-wave $\Xi_{b}^{\prime}$ against the parameter $b$. 
Given the difference $5 / 2$ in $\mathbf{L} \cdot \mathbf{S}_{Q}$ between the $5 / 2^{+}$and $3 / 2^{+}$states, one can extract $a_{2}$ for the $\Lambda_{c}(1 D)$ to have $a_{2}\left[\Lambda_{c}(1 D)\right]=\frac{2}{5}\left[\Lambda_{c}\left(5 / 2^{+}\right)-\Lambda_{c}\left(3 / 2^{+}\right)\right]=10.21 \mathrm{MeV}$.

The scaling relation (23) gives then

$$
a_{2}\left[\Lambda_{b}(1 D)\right]=a_{2}\left[\Lambda_{c}(1 D)\right]\left(\frac{1.44}{4.48}\right)=3.28 \mathrm{MeV},
$$

which predicts (in MeV), by Eq. (44),

$$
\begin{aligned}
M\left[\Lambda_{b}(1 D)\right] & =6149.3+3.28\left[\begin{array}{rr}
-3 / 2 & 0 \\
0 & 1
\end{array}\right] \\
& =\left[\begin{array}{rr}
6144.4 & 0 \\
0 & 6152.6
\end{array}\right],
\end{aligned}
$$

corresponding to $J=3 / 2^{+}, 5 / 2^{+}$, respectively. This is in good agreement with the observations.

Due to the lacking of the $B / B_{s}$ meson data for $D$-wave unfortunately, we are not able to test the scaling relation (24) directly with the SH mesons. An alternative is to verify it via scaling from the $D(1 D)$ mesons to the $\Lambda_{c}(1 D)$ and then to the $\Lambda_{b}(1 D)$. Combined with Eq. (22) and (C23) in Appendix C, Eq. (24) yields

$$
\begin{aligned}
a_{2}\left[\Lambda_{b}\right] & =a_{2}\left[\Lambda_{c}(1 D)\right]\left(\frac{M_{c}}{M_{b}}\right)=\frac{a_{2}[D(1 D)]}{1+m_{d} / M_{c}}\left(\frac{M_{c}}{M_{b}}\right) \\
& =\frac{12.6 \mathrm{MeV}}{1+0.535 / 1.44}\left(\frac{1.44}{4.48}\right)=2.95 \mathrm{MeV},
\end{aligned}
$$

which gives

$$
\begin{aligned}
M\left[\Lambda_{b}(1 D)\right] & =6149.27+\{-3 / 2,1\} * 2.95 \\
& =\{6144.8,6152.2\} \mathrm{MeV}
\end{aligned}
$$

This agrees with the LHCb observations equally well. The same procedure as in Eq. (60) applies for computing the $D$ wave masses of the $\Xi_{b}$, with the results collected in Table I.

Finally, we explain why the some of the four observed $\mathrm{SH}$ baryons discussed in this work can not be the lower $J^{P}=1 / 2^{-}$multiplet in the $P$-wave $\mathrm{SH}$ baryons so that they can be seen easily experimentally comparing with the $J^{P}=$ $3 / 2^{-}$multiplets. In the following, we choose $\Xi_{b}^{\prime}(6227)^{-}$ as a example to address this question briefly in the light of strong decay behavior which was not the main topics of this work. For more discussions on decays of the $\Sigma_{b}(6097) / \Xi_{b}^{\prime}(6227)^{-}$, see the literatures $[8-10,16]$.

First of all, we note that experiments by LHCb using $p p$ collision data found the $\Xi_{b}^{\prime}(6227)^{-}$to be relatively narrow $[39,40]$

$$
\Gamma\left[\Xi_{b}^{\prime}(6227)^{-}\right]=18.1 \pm 5.4 \pm 1.8 \mathrm{MeV},
$$

In principle the $1 / 2^{-}$assignment for the $\Xi_{b}^{\prime}(6227)^{-}$is allowed since in the $P$-wave the diquark angular momentum can be $j=1 \oplus 1=\{0,1,2\}$ and couple with $S_{Q}=1 / 2$ to give $J=1 / 2$. If this is the case, the decay to the $1 / 2^{+}$ground-state $\Xi_{b} \pi^{-}$(or $\Lambda_{b} K^{-}$) is very likely to happen via the $S$-wave by absorbing the $L=1$ orbital motion into the diquark system to flip the spin of one quark in diquark $b s^{\uparrow} q^{\uparrow}(1 P) \rightarrow b s^{\uparrow} q^{\downarrow}\left(1 S_{1 / 2}\right)+q^{\uparrow} \bar{q}^{\downarrow}$ (S-wave). This yields normally a large decay width, contradictory with the relatively narrow width of the $\Xi_{b}^{\prime}(6227)$ shown above. For the $J^{P}=3 / 2^{-} \mathrm{SH}$ baryons in $P$-wave, this kind of absorbing is prohibited by the angular momentum conservation, and the $P$-wave decay is also banned by the parity. So the $\Xi_{b}^{\prime}(6227)$ can decay strongly only via $D$ wave which agrees with its relatively narrow width due to the higher $L$ suppression. So, unlike the $1 / 2^{-}$state, a $3 / 2^{-}$ baryon $\Xi_{b}$ in $P$-wave is easier to be seen in experiments due to its relatively narrow width. In addition, though the $5 / 2^{-}$ assignment for the $\Sigma_{b}(6097)$ seems to be marginal, it cannot be excluded fully in the light of our mass analysis.

\section{SUMMARY AND DISCUSSIONS}

In this work we use Regge approach in the heavy quarkdiquark picture to re-examine the orbitally excited spectrum of the charmed and bottom baryons. Our analysis via a simple linear Regge relation (2) with the baryon mass shifted by the heavy quark mass $M \rightarrow M-M_{Q}$ indicates that the relation is able to describe the spin-averaged mass spectrum of the orbitally excited charmed and bottom baryons $\left(\Lambda_{Q}, \Xi_{Q}, \Sigma_{Q}\right.$ and $\left.\Xi_{Q}^{\prime}, Q=c, b\right)$. The further computations on spin-dependent mass splitting suggest that the baryons $\Xi_{b}(6227)^{-}$and the $\Sigma_{b}(6097)^{-}$, and the $\Sigma_{c}(2800)$ and the $\Xi_{c}^{\prime}(2930)$ are all the $1 P$-wave baryons with the spin-parity $J^{P}=3 / 2^{-}$preferably. We also present mass predictions of the unseen bottom baryon $\Xi_{b}$ in their $1 P$ and $1 D$ states, providing some useful clues for the coming experiments to find them, especially the LHCb.

It is noticeable that the relation (2) is derived from the classical relativistic energy and angular momentum of the rotating QCD string model, with no attempt made to obtain quantum correction to it. However, this relation shows the main feature predicted by the quantized theory of the QCD string $[32,33,41-43]$ : the linear functional form of trajectory remains intact, only with the intercept and the hadron mass shifted. When taking $L$ to be the quantized numbers, the approach in this work can be viewed, in a sense, as an leading semiclassical approximation of quantized theory of string. A recent quantum treatment of rotating string [33] in the nonvanishing endpoint-mass case yields a similar conclusion. We also note that for a string tied to one heavy and one light quarks, the mass shift $M \rightarrow M-M_{Q}$ in the relation (2) is crucial for describing the moderate nonlinear behavior of the baryon mass squared $M^{2}$ as a function of $L$ [41](see Figs. 6-7) at low- $L$ : such a nonlinearity is absorbed into Eq. (2) largely, since the latter takes the form: 


$$
M^{2}-2 M_{Q} M+M_{Q}^{2}=\pi a L+a_{0},
$$

when expanding it in $M_{Q}$. Obviously, a nonlinear dependence in $M^{2}$ happens in the left-hand side (LHS) of Eq. (61) which tends to vanish when $L$ is large. This is in consistent with the numerical computations in Ref. [41], and is supported by a systematic examination [25] for whole $\mathrm{SH}$ hadron systems (the $\mathrm{SH}$ mesons and baryons).

In order to compute the spin-dependent mass, we employed the scaling relations based on similarity in the color-configurations between a SH baryon and its meson partner or among the heavy flavor partners. While they are of approximate these relations turn out to be valuable as they have exhibited the most general features of the spindependent relativistic corrections, which scale as the magnetic moment $\mathbf{S}_{d} / m_{d}$ for the $a_{1}$-term and as $\mathbf{S}_{Q} / M_{Q}$ for the $a_{2}$-term in Eq. (9). We note that the color-structure similarity among these $\mathrm{SH}$ hadrons is implicitly assumed in the quark-diquark picture.

By the way, the following remarks are in order:

(1) Without adding or tuning of the parameters, a simple application of our computational procedure to the $D$ wave bottom baryon $\Lambda_{b}$ yields a spin-averaged mass and spin-dependent mass splitting which agree well with the observed spin-averaged mass $6149.97 \mathrm{MeV}$ of the newly reported resonances $\Lambda_{b}$ [38] and its mass splitting $-3.80 \mathrm{MeV}$ and $2.54 \mathrm{MeV}$ for the $3 / 2^{+}$and $5 / 2^{+}$states, respectively.

(2) By predicting spin-averaged masses, the relation (2) are helpful in itself in identifying the state order of the excited spin-multiplets in the sense of the bisection of the mass intervals. To accommodate them further, detailed computations about the mass differences of the spin-multiplets are necessary.

(3) Similar decay behaviors (the pseudoscalar meson emission and relatively narrow widths) seen for the baryons $\Xi_{b}(6227)^{-} / \Sigma_{b}(6097)^{-}$, the $\Sigma_{c}(2800) /$ $\Xi_{c}^{\prime}(2930)$ prefer to support that they are all the $1 P$-wave baryons with $J^{P}=3 / 2^{-}$. Further exploring of the parameter $b$ for them becomes valuable for decoding inner-structures of the $P$-wave partners of these baryons.

(4) The method in this work can in principle be used to estimate the higher orbital excitations of the bottom baryons, with the experimental mass information of the excited meson partners or the charmed partners increasing. To go beyond the limitation having to refer to the SH meson mass spectrum in computing the mass splitting, the quantized string dynamics is desirable for further exploring the heavy baryon spectroscopy.

\section{ACKNOWLEDGMENTS}

D. J. thanks H-X. Chen for useful discussions. D. J. is supported by the National Natural Science Foundation of China under the Grant No. 11565023 and the Feitian
Distinguished Professor Program of Gansu. D. J. and A. H. are supported in part by Grant-in-Aid for Scientific Research on Innovative Areas, "Clustering as a window on the hierarchical structure of quantum systems."

Note added.-After submission of this work, two newly reported resonances $\Lambda_{b}(6146)^{0}$ and the $\Lambda_{b}(6146)^{0}$ [38] come to our attention, which can be nicely accommodated as the $D$-wave singly bottom baryons using our approach in this work. The agreement of our prediction with the observed mass data is remarkable.

\section{APPENDIX A}

For the orbitally excitations, the classical energy and orbital angular momentum for the rotating QCD string read $[28,41,44]$

$$
\begin{aligned}
& E=\frac{m_{Q}}{\sqrt{1-v_{Q}^{2}}}+\frac{m}{\sqrt{1-v_{d}^{2}}}+\frac{a}{\omega} \sum_{i=Q, d} \int_{0}^{v_{i}} \frac{d u}{\sqrt{1-u^{2}}}, \\
& L=\frac{m_{Q} v_{Q}^{2} / \omega}{\sqrt{1-v_{Q}^{2}}}+\frac{m v_{d}^{2} / \omega}{\sqrt{1-v_{d}^{2}}}+\frac{a}{\omega^{2}} \sum_{i=Q, d} \int_{0}^{v_{i}} \frac{u^{2} d u}{\sqrt{1-u^{2}}},
\end{aligned}
$$

where $m_{Q}$ and $m$ are the bare masses of the heavy quark $Q$ and light diquarks $d$ and $v_{i}=\omega r_{i}(i=Q, d)$ the velocity of the string end tied to the quark $i=Q, d$. It is known that the Selem-Wilczek relation (4) follows from upon the $\left(m_{i} \omega / a\right)$-expansion of (A1) and (A2) [28]. Following [27], we define the effective (dynamical) masses of the heavy quark and the light diquark in the $\mathrm{CM}$ frame of the baryon by

$$
M_{Q}=\frac{m_{Q}}{\sqrt{1-v_{Q}^{2}}}, \quad m_{d}=\frac{m}{\sqrt{1-v_{d}^{2}}},
$$

to rewrite (A1) and (A2) as

$$
\begin{aligned}
E=M_{Q} & +m_{d}+\frac{a}{\omega}\left[\arcsin \left(v_{d}\right)+\arcsin \left(v_{Q}\right)\right], \\
L= & \frac{1}{\omega}\left(M_{Q} v_{Q}^{2}+m_{d} v_{d}^{2}\right) \\
& +\frac{a}{2 \omega^{2}} \sum_{i=Q, d}\left[\arcsin \left(v_{i}\right)-v_{i} \sqrt{1-v_{i}^{2}}\right],
\end{aligned}
$$

in which the last term in (A4) is the contribution to the orbital angular momentum due to the string rotating.

The boundary condition of string at ends with heavy quark gives

$$
\frac{a}{\omega}=\frac{m_{Q} v_{Q}}{1-v_{Q}^{2}}=\frac{M_{Q} v_{Q}}{\sqrt{1-v_{Q}^{2}}},
$$

or 


$$
\frac{a}{\omega} \simeq P_{Q}+\frac{P_{Q}^{3}}{2 M_{Q}^{2}},
$$

with $P_{Q} \equiv M_{Q} v_{Q}$ the conserved momentum of the heavy quark. Expanding Eqs. (A3) and (A4) in $v_{Q}$ and $m$ gives, up to $v_{Q}^{4}$ and the leading order of $m$,

$$
\begin{aligned}
& E=M_{Q}+m_{d}+\frac{\pi a}{2 \omega}+\frac{a}{\omega}\left[v_{Q}-\frac{m}{m_{d}}+\frac{1}{6} v_{Q}^{3}\right]+\mathcal{O}\left[v_{Q}^{5}\right], \\
& \omega L=m_{d}+M_{Q} v_{Q}^{2}+\frac{a}{\omega}\left[\frac{\pi}{4}-\frac{m}{m_{d}}\right]+\frac{a}{3 \omega} v_{Q}^{3}+\mathcal{O}\left[v_{Q}^{5}\right] .
\end{aligned}
$$

Using Eq. (A7) and upon eliminating $\omega$, Eqs. (A8)-(A9) combines to give, when ignoring the small term $m / m_{d}$,

$$
\left(E-M_{Q}\right)^{2}=\pi a L+\left(m_{d}+\frac{P_{Q}^{2}}{M_{Q}}\right)^{2}-2 m P_{Q} .
$$

This leads to Eq. (2) when taking the bare mass $m \rightarrow 0$. By the way, we note that Eq. (3) follows by rewriting the velocity $v_{Q}^{2}=1-\left(m_{Q} / M_{Q}\right)^{2}$, with $m_{Q} \equiv m_{\text {bare } Q}$ the bare mass of the heavy quark $Q$.

Note that the heavy quark limit(HQL) is defined as the largeness of the bare mass $m_{Q}$ of the heavy quark $Q$ entering the QCD Lagrangian while its 3-momentum $P_{Q}=$ $M_{Q} v_{Q}$ remains fixed as $\mathrm{Q}$ behaves like a static external source [29] within the bound state of hadrons. It follows from Eq. (A3) that in the HQL the effective mass $M_{Q}$ tends to infinity also while the 3 -velocity $v_{Q}$ tends to vanish, that is, the heavy quarks move nonrelativistically within hadrons, as it should be. One can readily check that in the HQL, Eqs. (A8) and (A9) become $E=M_{Q}+m_{d}+\frac{\pi a}{2 \omega}$ and $\omega L=m_{d}+\frac{a \pi}{4 \omega}$, which yield $\left(E-M_{Q}\right)^{2}=\pi a L+m_{d}^{2}$. Here, $M_{Q} v_{Q}^{2}=P_{Q}^{2} / M_{Q}$ tends to vanish in the right-hand side (RHS) of Eq. (A9).

\section{APPENDIX B}

For the heavy quark-diquark systems, the matrix elements of $\mathbf{L} \cdot \mathbf{S}_{i}$ can be calculated by explicit construction of the baryon states with a given $J_{3}$ as linear combinations of the states $\left|S_{d 3}, S_{Q 3}, L_{3}\right\rangle$ with $S_{d 3}+S_{Q 3}+L_{3}=J_{3}$. Due to the rotation invariance of the matrix elements, it suffices to use a single $J_{3}$ for each and, one can use

$$
\mathbf{L} \cdot \mathbf{S}_{i}=\frac{1}{2}\left[L_{+} S_{i-}+L_{-} S_{i+}\right]+L_{3} S_{i 3},
$$

to find their elements by applying $\mathbf{L} \cdot \mathbf{S}_{i}$ on the third components of angular momenta. In the rep. of $\left|L_{z}, S_{Q z}, S_{d z}\right\rangle$ these component are given by the following basis states [35]

$$
\begin{aligned}
\left|1^{2} P_{1 / 2}, J_{z}=1 / 2\right\rangle= & \frac{\sqrt{2}}{3}|1,-1 / 2,0\rangle-\frac{1}{3}|0,1 / 2,0\rangle \\
& -\frac{\sqrt{2}}{3}|0,-1 / 2,1\rangle+\frac{2}{3}|-1,1 / 2,1\rangle, \\
\left|1^{4} P_{1 / 2}, J_{z}=1 / 2\right\rangle= & \frac{1}{\sqrt{2}}|1,1 / 2,-1\rangle-\frac{1}{3}|1,-1 / 2,0\rangle \\
& -\frac{\sqrt{2}}{3}|0,1 / 2,0\rangle+\frac{1}{3}|0,-1 / 2,1\rangle \\
& +\frac{1}{3 \sqrt{2}}|-1,1 / 2,1\rangle, \\
\left|1^{2} P_{3 / 2}, J_{z}=3 / 2\right\rangle= & \sqrt{\frac{2}{3}}|1,-1 / 2,1\rangle-\sqrt{\frac{1}{3}}|0,1 / 2,1\rangle, \\
\left|1^{4} P_{3 / 2}, J_{z}=3 / 2\right\rangle= & \sqrt{\frac{3}{5}}|1,1 / 2,0\rangle-\sqrt{\frac{2}{15}}|1,-1 / 2,1\rangle \\
& -\frac{2}{\sqrt{15}}|0,1 / 2,1\rangle, \\
\mid 1^{4} P_{5 / 2}, J_{z} & =5 / 2\rangle=|1,1 / 2,1\rangle .
\end{aligned}
$$

Having these component states (in $L-S$ coupling), one can compute the matrix elements of $\mathbf{L} \cdot \mathbf{S}_{d}$,

$$
\begin{aligned}
& \left\langle\mathbf{L} \cdot \mathbf{S}_{d}\right\rangle_{J=\frac{1}{2}}=\left[\begin{array}{rc}
-\frac{4}{3} & -\frac{\sqrt{2}}{3} \\
-\frac{\sqrt{2}}{3} & -\frac{5}{3}
\end{array}\right], \\
& \left\langle\mathbf{L} \cdot \mathbf{S}_{d}\right\rangle_{J=\frac{3}{2}}=\left[\begin{array}{rc}
\frac{2}{3} & -\frac{\sqrt{5}}{3} \\
-\frac{\sqrt{5}}{3} & -\frac{2}{3}
\end{array}\right] .
\end{aligned}
$$

Accordingly, the states in terms of the $J-j$ coupling can be expressed as linear combinations of states with definite $L-S$ coupling (for each eigenvalue $\lambda$ of $\mathbf{L} \cdot \mathbf{S}_{d}$.):

$$
\begin{aligned}
& \lambda=-2:\left|J=\frac{1}{2}, j=0\right\rangle=\sqrt{\frac{1}{3}}\left|1^{2} P_{1 / 2}\right\rangle+\sqrt{\frac{2}{3}}\left|1^{4} P_{1 / 2}\right\rangle, \\
& \lambda=-1:\left|J=\frac{1}{2}, j=1\right\rangle=\sqrt{\frac{2}{3}}\left|1^{2} P_{1 / 2}\right\rangle-\sqrt{\frac{1}{3}}\left|1^{4} P_{1 / 2}\right\rangle, \\
& \lambda=-1:\left|J=\frac{3}{2}, j=1\right\rangle=\sqrt{\frac{1}{6}}\left|1^{2} P_{3 / 2}\right\rangle+\sqrt{\frac{5}{6}}\left|1^{4} P_{3 / 2}\right\rangle,
\end{aligned}
$$

$$
\lambda=+1:\left|J=\frac{3}{2}, j=2\right\rangle=\sqrt{\frac{5}{6}}\left|1^{2} P_{3 / 2}\right\rangle-\sqrt{\frac{1}{6}}\left|1^{4} P_{3 / 2}\right\rangle,
$$




$$
\lambda=+1:\left|J=\frac{5}{2}, j=2\right\rangle=\left|1^{4} P_{5 / 2}\right\rangle,
$$

which can be used to average $\mathbf{L} \cdot \mathbf{S}_{Q}$ and $\mathbf{S}_{12}$. The detailed results are collected in Table X.

\section{APPENDIX C}

We describe here some of the details on how to estimate the values of the parameters $a_{1}, a_{2}, b, c$ that enter the spindependent interaction (9) for the $\mathbf{S H}$ mesons where $\mathbf{S}_{d}$ is replaced everywhere in Appendix $\mathrm{C}$ by the spin $\mathbf{S}_{q}$ of light quark. The following three cases of excited states are considered in order:

(1) The S-wave $\mathrm{SH}$ mesons. In this ground-state case, only the contact term $c \mathbf{S}_{Q} \cdot \mathbf{S}_{q}$ remains in the spindependent interaction (9). Since the expectation values of $\mathbf{S}_{Q} \cdot \mathbf{S}_{q}$ are $-3 / 4$ and $1 / 4$ in the $1^{1} S_{0}$ and $1^{3} S_{1}$ meson states, respectively, the parameter $c$ can be estimated simply by confronting the predicted mass difference between two S-states, $c(1 / 4+3 / 4)=c$, with the measured $S$-wave masses of the $D$ mesons in the Table VII. The result is

$$
c=(2010.3-1869.7) \mathrm{MeV}=140.6 \mathrm{MeV} .
$$

(2) The $P$-wave $\mathrm{SH}$ mesons. Similar to Appendix B, a calculation can be performed for the $P$-wave $\mathrm{SH}$ mesons. As in the case of the SH baryons, we find it convenient to work in the $j$-j basis in which the analogue of the first term in Eq. (9), $a_{1} \mathbf{L} \cdot \mathbf{S}_{q}$, is diagonal. We first calculate the expectation values of $\mathbf{L} \cdot \mathbf{S}$ and the tensor operator $\mathbf{S}_{12}$ in the basis states ${ }^{2 S+1} P_{J}$. The results are shown in Table XI.

To evaluate the expectation values of $\mathbf{L} \cdot \mathbf{S}_{i}(i=q, Q)$, it is convenient to use the $L-S$ basis. When labeling the states by $\left|S_{q 3}, S_{Q 3}, L_{3}\right\rangle$, one can construct the $L-S$ basis by

$$
\begin{gathered}
\left|{ }^{3} P_{0}, J_{3}=0\right\rangle=\left|-\frac{1}{2},-\frac{1}{2}, 1\right\rangle, \quad \text { (C2) } \\
\left|{ }^{1} P_{1}, J_{3}=1\right\rangle=\frac{1}{\sqrt{2}}\left|\frac{1}{2},-\frac{1}{2}, 1\right\rangle-\frac{1}{\sqrt{2}}\left|-\frac{1}{2}, \frac{1}{2}, 1\right\rangle, \\
\left|{ }^{3} P_{1}, J_{3}=1\right\rangle=\frac{1}{2}\left|-\frac{1}{2}, \frac{1}{2}, 1\right\rangle+\frac{1}{2}\left|\frac{1}{2},-\frac{1}{2}, 1\right\rangle \\
-\frac{1}{\sqrt{2}}\left|\frac{1}{2}, \frac{1}{2}, 0\right\rangle, \\
\left|{ }^{3} P_{2}, J_{3}=2\right\rangle=\left|\frac{1}{2}, \frac{1}{2}, 1\right\rangle .
\end{gathered}
$$

TABLE XI. Expectation values of the spin-dependent terms for $P$-wave $c \bar{q}$ mesons in the $L-S$-coupling basis states.

\begin{tabular}{lccc}
\hline \hline State & $\langle\mathbf{L} \cdot \mathbf{S}\rangle$ & $\left\langle\mathbf{S}_{12}\right\rangle$ & $\left\langle\mathbf{S}_{Q} \cdot \mathbf{S}_{q}\right\rangle$ \\
\hline${ }^{3} P_{0}$ & -2 & -4 & $1 / 4$ \\
${ }^{1} P_{1}$ & 0 & 0 & $-3 / 4$ \\
${ }^{3} P_{1}$ & -1 & 2 & $1 / 4$ \\
${ }^{3} P_{2}$ & 1 & $-2 / 5$ & $1 / 4$ \\
\hline \hline
\end{tabular}

Using (B1) in the $J=1$ subspace given by the basis $\left({ }^{1} P_{1},{ }^{3} P_{1}\right)$, the matrices describing mixing of the $J=1$ states are

$$
\begin{gathered}
\left\langle\mathbf{L} \cdot \mathbf{S}_{q}\right\rangle_{J=1}=\left[\begin{array}{rr}
0 & 1 / \sqrt{2} \\
1 / \sqrt{2} & -1 / 2
\end{array}\right], \\
\left\langle\mathbf{L} \cdot \mathbf{S}_{Q}\right\rangle_{J=1}=\left[\begin{array}{rr}
0 & -1 / \sqrt{2} \\
-1 / \sqrt{2} & -1 / 2
\end{array}\right] .
\end{gathered}
$$

The first matrix, which has the eigenvalues -1 and $1 / 2$, can be diagonalized by transforming the $L-S$ eigenstates to the $|J, j\rangle$ eigenstates. This transformation, when written in the whole space, can be given by

$$
\begin{aligned}
& |J=0, j=1 / 2\rangle=\left|{ }^{3} P_{0}\right\rangle, \\
& \left.|J=1, j=1 / 2\rangle=\sqrt{\frac{1}{3}}\left|{ }^{1} P_{1}\right\rangle-\left.\sqrt{\frac{2}{3}}\right|^{3} P_{1}\right\rangle, \\
& \left.|J=1, j=3 / 2\rangle=\sqrt{\frac{2}{3}}\left|{ }^{1} P_{1}\right\rangle+\left.\sqrt{\frac{1}{3}}\right|^{3} P_{1}\right\rangle, \\
& |J=2, j=3 / 2\rangle=\left|{ }^{3} P_{2}\right\rangle .
\end{aligned}
$$

With the expressions above one can work out the expectation values of all spin-dependent operators contributing to the masses of the $P$-wave $Q \bar{q}$ mesons and thereby obtain estimations for the spin-dependent mass splitting via expanding around the eigenstates of $\mathbf{j}=\mathbf{L}+\mathbf{S}_{q}$. Including the spin-averaged mass $\bar{M}$, the results for the $P$-wave are [35],

$$
\begin{array}{r}
M(0,1 / 2)=\bar{M}-a_{1}-a_{2}-b-\frac{c}{4}, \\
M(1,1 / 2)=\bar{M}-a_{1}+\frac{1}{3} a_{2}+\frac{1}{3} b-\frac{c}{12}, \\
M(1,3 / 2)=\bar{M}+\frac{1}{2} a_{1}-\frac{5}{6} a_{2}+\frac{1}{6} b-\frac{5 c}{12}, \\
M(2,3 / 2)=\bar{M}+\frac{1}{2} a_{1}+\frac{1}{2} a_{2}-\frac{1}{10} b+\frac{c}{4} .
\end{array}
$$


Given Eqs. (C8)-(C11), the parameters $\left(a_{1}, a_{2}, b\right)$ which reproduce the observed masses of the $c \bar{s}$ mesons in Table VII are [35]

$\left\{\begin{array}{cccc}\bar{M}(\mathrm{MeV}), & a_{1}(\mathrm{MeV}), & a_{2}(\mathrm{MeV}) & b(\mathrm{MeV}) \\ 2513.4, & 89.4, & 40.7, & 65.6,\end{array}\right\}$

(C12)

where the parameter $c$ in the hyperfine term is taken to be zero as it is quite small in the $P$-wave state.

(3) The $D$-wave $\mathrm{SH}$ mesons. The similar procedure applies for the $D$-wave $Q \bar{q}$ mesons. Using the state $\left|S_{q 3}, S_{Q 3}, L_{3}\right\rangle$, one can explicitly construct the $L-S$ basis for the $D$-wave $Q \bar{q}$ states by

$$
\begin{aligned}
\left|{ }^{3} D_{1}, J_{3}=1\right\rangle & =\sqrt{\frac{3}{5}}\left|-\frac{1}{2},-\frac{1}{2}, 2\right\rangle+\sqrt{\frac{1}{10}}\left|\frac{1}{2}, \frac{1}{2}, 0\right\rangle \\
& -\sqrt{\frac{3}{20}}\left|\frac{1}{2},-\frac{1}{2}, 1\right\rangle-\sqrt{\frac{3}{20}}\left|-\frac{1}{2}, \frac{1}{2}, 1\right\rangle,
\end{aligned}
$$

$$
\left|{ }^{1} D_{2}, J_{3}=2\right\rangle=\frac{1}{\sqrt{2}}\left|\frac{1}{2},-\frac{1}{2}, 2\right\rangle-\frac{1}{\sqrt{2}}\left|-\frac{1}{2}, \frac{1}{2}, 2\right\rangle,
$$

$$
\begin{aligned}
\left|{ }^{3} D_{2}, J_{3}=2\right\rangle= & -\frac{1}{\sqrt{3}}\left|\frac{1}{2}, \frac{1}{2}, 1\right\rangle+\frac{1}{\sqrt{3}}\left|\frac{1}{2},-\frac{1}{2}, 2\right\rangle \\
& +\frac{1}{\sqrt{3}}\left|-\frac{1}{2}, \frac{1}{2}, 2\right\rangle, \quad
\end{aligned}
$$

$$
\left|{ }^{3} D_{3}, J_{3}=3\right\rangle=\left|\frac{1}{2}, \frac{1}{2}, 2\right\rangle .
$$

Utilizing (B1) again in the $J=2$ subspace given by the basis $\left({ }^{1} D_{2},{ }^{3} D_{2}\right)$, the matrices describing mixing of the $J=2$ states are

$$
\begin{gathered}
\left\langle\mathbf{L} \cdot \mathbf{S}_{q}\right\rangle_{J=2}=\left[\begin{array}{rr}
0 & \sqrt{3 / 2} \\
\sqrt{3 / 2} & -1 / 2
\end{array}\right], \\
\left\langle\mathbf{L} \cdot \mathbf{S}_{Q}\right\rangle_{J=2}=\left[\begin{array}{rr}
0 & -\sqrt{3 / 2} \\
-\sqrt{3 / 2} & -1 / 2
\end{array}\right] .
\end{gathered}
$$

The first matrix, which has the eigenvalues $-3 / 2$ and 1 , can be diagonalized by the following basis transformation between the $L-S$ eigenstates and the $|J, j\rangle$ eigenstates. In the whole space, the transformation takes the form:

$$
\begin{gathered}
|J=1, j=3 / 2\rangle=\left|{ }^{3} D_{1}\right\rangle, \\
|J=2, j=3 / 2\rangle=-\sqrt{\frac{2}{5}}\left|{ }^{1} P_{2}\right\rangle-\sqrt{\frac{3}{5}}\left|{ }^{3} P_{2}\right\rangle, \\
\left.|J=2, j=5 / 2\rangle=\sqrt{\frac{3}{5}}\left|{ }^{1} P_{2}\right\rangle+\left.\sqrt{\frac{2}{5}}\right|^{3} P_{2}\right\rangle, \\
|J=3, j=5 / 2\rangle=\left|{ }^{3} P_{3}\right\rangle .
\end{gathered}
$$

Given these relations, the calculations for the expectation values of all mass splitting operators can be performed and the mass expression for the $D$-wave $Q \bar{q}$ mesons can be obtained via the procedure similar to that to have Eqs. (16)(20). The results are

$$
\begin{array}{r}
M(1,3 / 2)=\bar{M}-\frac{3}{2} a_{1}-\frac{3}{2} a_{2}-\frac{5}{2} b+\frac{c}{4}, \\
M(2,3 / 2)=\bar{M}-\frac{3}{2} a_{1}+\frac{9}{10} a_{2}+\frac{3}{2} b-\frac{3 c}{2}, \\
M(2,5 / 2)=\bar{M}+a_{1}-\frac{7}{5} a_{2}+\frac{7}{5} b-\frac{7 c}{20}, \\
M(3,5 / 2)=\bar{M}+a_{1}+a_{2}-b-\frac{c}{4}
\end{array}
$$

In the $D$-wave case, only two observed masses are available in Table VII for each family of the $D / D_{s}$ mesons. Since only the mass splitting $\Delta M=M-\bar{M}$ is relevant here, we use the mass splitting $\Delta M$ given by

TABLE XII. The masses and corresponding mass splitting $\Delta M=M-\bar{M}$ for the $D$-wave $D$ mesons. The QM stands for the quark model predictions $(\bar{M}=2834.3 \mathrm{MeV})$. The rescaled mass splitting are obtained by multiplying $\Delta M(\mathrm{QM})$ by a deviation factor $(2763.5-2737) /(2863-2850)=2.04$.

\begin{tabular}{lcccc}
\hline \hline States & $M($ Exp. $)$ & $M(\mathrm{QM})[34]$ & $\Delta M(\mathrm{QM})$ & $\Delta M$ (rescaled) \\
\hline$|1,3 / 2\rangle$ & $\ldots$ & 2788 & -46.3 & -94.4 \\
$|2,3 / 2\rangle$ & $\cdots$ & 2806 & -28.3 & -57.6 \\
$|2,5 / 2\rangle$ & $2737(12)$ & 2850 & 15.8 & 32.1 \\
$|3,5 / 2\rangle$ & 2763.5 & 2863 & 28.8 & 58.7 \\
\hline \hline
\end{tabular}


the quark model $(\mathrm{QM})$ predictions [34] rescaled by the deviation factor 2.04 so that the rescaled mass splitting match the observed data. The details are listed in Table XII.

The parameters for the $D$-wave $D$ mesons which reproduce the mass splitting $\Delta M$ (rescaled) in Table IX are

$$
\left\{\begin{array}{ccc}
a_{1}(\mathrm{MeV}), & a_{2}(\mathrm{MeV}) & b(\mathrm{MeV}) \\
47.6, & 12.6, & 0.52,
\end{array}\right\}
$$

where the parameter $c$ in the hyperfine term has been set to be zero. This completes the estimation of $a_{2}$ for the SH mesons in their $P$-wave and $D$-wave excitations.
[1] N. Isgur and M. B. Wise, Spectroscopy with Heavy Quark Symmetry, Phys. Rev. Lett. 66, 1130 (1991).

[2] M. Tanabashi et al. (Particle Data Group), Review of particle physics, Phys. Rev. D 98, 030001 (2018).

[3] R. Aaij et al. (LHCb Collaboration), Observation of a New $\Xi_{b}^{-}$Resonance, Phys. Rev. Lett. 121, 072002 (2018).

[4] R. Aaij et al. (LHCb Collaboration), Observation of Two Resonances in the $\Lambda_{b}^{0} \pi^{ \pm}$Systems and Precise Measurement of $\Sigma_{b}^{ \pm}$and $\Sigma_{b}^{* \pm}$ b Properties, Phys. Rev. Lett. 122, 012001 (2019).

[5] T. M. Aliev, K. Azizi, Y. Sarac, and H. Sundu, Structure of the $\Xi_{b}^{-}(6227)$ resonance, Phys. Rev. D 98, 094014 (2018).

[6] B. Chen, K. W. Wei, X. Liu, and A. Zhang, Role of newly discovered $\Xi_{b}^{-}(6227)$ for constructing excited bottom baryon family, Phys. Rev. D 98, 031502(R) (2018).

[7] B. Chen and X. Liu, Assigning the newly reported $\Sigma_{b}(6097)$ as a $P$-wave excited state and predicting its partners, Phys. Rev. D 98, 074032 (2018).

[8] P. Yang, J. J. Guo, and A. Zhang, Identification of the newly observed $\Sigma_{b}(6097)^{ \pm}$baryons from their strong decays, Phys. Rev. D 99, 034018 (2019).

[9] K.-L. Wang, Q.-F. Lü, and X.-H. Zhong, Interpretation of the newly observed $\Sigma_{b}(6097)^{-}$and $\Xi_{b}(6227)^{-}$states as the $P$-wave bottom baryons, Phys. Rev. D 99, 014011 (2019).

[10] E. L. Cui, H. M. Yang, H.X. Chen, and A. Hosaka, Identifying the $\Xi_{b}(6227)$ and $\Sigma_{b}(6097)$ as $P$-wave bottom baryons of $J^{P}=3 / 2^{-}$, Phys. Rev. D 99, 094021 (2019).

[11] T. M. Aliev, K. Azizi, Y. Sarac, and H. Sundu, Determination of the quantum numbers of $\Sigma_{b}(6097)^{ \pm}$via their strong decays, Phys. Rev. D 99, 094003 (2019).

[12] Y. Huang, C. J. Xiao, L. S. Geng, and J. He, Strong decays of the $\Xi_{b}(6227)$ as a $\Sigma_{b} \bar{K}$ molecule, Phys. Rev. D 99, 014008 (2019).

[13] J. G. Körner, M. Kramer, and D. Pirjol, Heavy baryons, Prog. Part. Nucl. Phys. 33, 787 (1994).

[14] V. Crede and W. Roberts, Progress towards understanding baryon resonances, Rep. Prog. Phys. 76, 076301 (2013).

[15] H. Y. Cheng, Charmed baryons circa 2015, Front. Phys. 10, 101406 (2015).

[16] Q. Mao, H. X. Chen, W. Chen, A. Hosaka, X. Liu, and S. L. Zhu, QCD sum rule calculation for $P$-wave bottom baryons, Phys. Rev. D 92, 114007 (2015).

[17] D. Ebert, R. N. Faustov, and V. O. Galkin, Spectroscopy and Regge trajectories of heavy baryons in the relativistic quarkdiquark picture, Phys. Rev. D 84, 014025 (2011).
[18] D. Ebert, R. N. Faustov, and V. O. Galkin, Masses of excited heavy baryons in the relativistic quark-diquark picture, Phys. Lett. B 659, 612 (2008).

[19] E. Klempt and J. M. Richard, Baryon spectroscopy, Rev. Mod. Phys. 82, 1095 (2010).

[20] W. Roberts and M. Pervin, Heavy baryons in a quark model, Int. J. Mod. Phys. A 23, 2817 (2008).

[21] B. Aubert et al. (BABAR Collaboration), Measure Bments of $\mathscr{B}\left(\bar{B}^{0} \rightarrow \wedge_{c}^{+} \bar{p}\right)$ and studies of $\mathscr{B}\left(B^{-} \rightarrow \wedge_{c}^{+} \bar{p} \pi^{-}\right)$resonances, Phys. Rev. D 78, 112003 (2008).

[22] Y. B. Li et al. (Belle Collaboration), Evidence of a structure in $\bar{K}^{0} \wedge_{c}^{+}$consistent with a charged $\Xi_{c}(2930)^{+}$, and updated measurement of $\bar{B}^{0} \rightarrow \bar{K}^{0} \wedge_{c}^{+} \bar{\Lambda}_{c}^{-}$at Belle, Eur. Phys. J. C 78, 928 (2018).

[23] H. Y. Cheng and C. W. Chiang, Quantum numbers of $\Omega_{c}$ states and other charmed baryons, Phys. Rev. D 95, 094018 (2017).

[24] T. Regge, Introduction to complex orbital momenta, Nuovo Cimento 14, 951 (1959).

[25] K. Chen, Y. Dong, X. Liu, Q. F. Lü, and T. Matsuki, Reggelike relation and a universal description of heavy-light systems, Eur. Phys. J. C 78, 20 (2018).

[26] H.-X. Chen, W. Chen, X. Liu, Y.-R. Liu, and S.-L. Zhu, A review of the open charm and open bottom systems, Rep. Prog. Phys. 80, 076201 (2017).

[27] B. Chen, K. W. Wei, and A. Zhang, Assignments of $Q$ and $Q$ baryons in the heavy quark-light diquark picture, Eur. Phys. J. A 51, 82 (2015).

[28] A. Selem and F. Wilczek, Hadron systematics and emergent diquarks, from fields strings: Circumnavig, Theor. Phys 1, 377 (2006).

[29] A. V. Manohar and M. B. Wise, Heavy Quark Physics (Cambridge University Press, Cambridge, England, 2010).

[30] L. A. Copley, N. Isgur, and G. Karl, Charmed baryons in a quark model with hyperfine interactions, Phys. Rev. D 20, 768 (1979); Erratum, Phys. Rev. D 23, 817 (1981).

[31] H. Nagahiro, S. Yasui, A. Hosaka, M. Oka, and H. Noumi, Structure of charmed baryons studied by pionic decays, Phys. Rev. D 95, 014023 (2017).

[32] C. Rebbi, Dual models and relativistic quantum strings, Phys. Rep. 12, 1 (1974).

[33] J. Sonnenschein and D. Weissman, Quantizing the rotating string with massive endpoints, J. High Energy Phys. 06 (2018) 148. 
[34] D. Ebert, R. N. Faustov, and V. O. Galkin, Heavy-light meson spectroscopy and Regge trajectories in the relativistic quark model, Eur. Phys. J. C 66, 197 (2010).

[35] M. Karliner and J. L. Rosner, Prospects for observing the lowest-lying odd-parity $\Sigma_{c}$ and $\Sigma_{b}$ baryons, Phys. Rev. D 92, 074026 (2015).

[36] M. Karliner and J. L. Rosner, Very narrow excited $\Omega_{c}$ baryons, Phys. Rev. D 95, 114012 (2017).

[37] R. N. Cahn and D. Jackson, Spin-orbit and tensor forces in heavy-quark light-quark mesons: Implications of the new $D_{s}$ state at $2.32 \mathrm{GeV}$, Phys. Rev. D 68, 037502 (2003).

[38] LHCb Collaboration, Observation of New Resonances in the $\Lambda_{b}^{0} \pi^{+} \pi^{-}$System, Phys. Rev. Lett. 123, 152001 (2019).

[39] R. Aaij et al. (LHCb Collaboration), Observation of Two Resonances in the $\Lambda_{b}^{0} \pi^{ \pm}$Systems and Precise Measurement of $\Sigma_{b}^{ \pm}$and $\Sigma_{b}^{* \pm}$ Properties, Phys. Rev. Lett. 122, 012001 (2019).

[40] R. Aaij et al. (LHCb Collaboration), Observation of a New $\Xi_{b}^{-}$Resonance, Phys. Rev. Lett. 121, 072002 (2018).

[41] D. LaCourse and M. G. Olsson, The string potential model. 1. spinless quarks, Phys. Rev. D 39, 2751 (1989).

[42] M. Baker and R. Steinke, Semiclassical quantization of effective string theory and Regge trajectories, Phys. Rev. D 65, 094042 (2002).

[43] T. J. Allen, C. Goebel, M. G. Olsson, and S. Veseli, Analytic quantization of the QCD string, Phys. Rev. D 64, 094011 (2001).

[44] K. Johnson and C. Nohl, Simple semiclassical model for the rotational states of mesons containing massive quarks, Phys. Rev. D 19, 291 (1979). 